\title{
High Frequency Trading und Verantwortung für systemische Risiken am Finanzmarkt
}

\section{Einleitung}

Im Rahmen des Gesamtprojekts «Krise der Zukunft» beschäftigt sich der nachfolgende Beitrag mit krisenhaften Szenarien auf den Finanzmärkten. Er widmet sich der Frage, ob es so etwas wie eine Apokalypse - ein Ende der Märkte - geben könnte, und wenn ja, wie man die Risiken eines solchen Ereignisses einschätzen bzw. wie man mit diesen Risiken umgehen kann. Der Fokus der Untersuchung liegt dabei auf der Verbreitung der computergesteuerten Handelsprogramme, ihrem Zerstörungspotenzial für die Finanzmärkte und dem ethischen Umgang mit den entsprechenden Risiken, vor allem der Frage nach der Verantwortung.

Nach der letzten Finanzkrise wurden Stimmen laut, dass die Finanzmärkte grundsätzlich krisenanfälliger geworden sind, sodass man sogar mit einem radikalen Kollaps rechnen müsste: Im September 2008 stand man wohl kurz vor solch einem dramatischen Ereignis. Dabei wurde auch erkannt, dass eine Finanzkrise nicht nur die unmittelbaren Marktteilnehmer betrifft, sondern auch viel breitere ökonomische Kreise und die Gesellschaft insgesamt: Ersparnisse, Altersvorsorge, Arbeitsplätze und manchmal elementare Versorgung mit allem Lebensnotwendigen gerieten in vielen Ländern in Gefahr. Die Arbeitslosigkeit der Jugendlichen in Spanien von über $40 \%$ im Jahr 2010 stellte einen solchen unerwünschten Effekt dar. Aus dieser Perspektive betrachtet ist die Frage nach dem reibungslosen Funktionieren der Finanzmärkte eine Frage der Risikoethik: Wer sind die Urheber der Risiken eines radikalen Marktkollapses und wer sind die Betroffenen? Wie viele Risiken könnte man wem zumuten? Wer trägt die Verantwortung dafür, dass Märkte kollabieren können (prospektiv), sowie im Falle, dass diese Risiken sich materialisieren (retrospektiv)?

Bevor man zur Beantwortung dieser Fragen übergeht, muss geklärt werden, um welche Art von Risiken es sich genau handelt. Bis zu der Krise 2008 hat man dem Risiko der Marktzerstörung wenig Beachtung geschenkt. Aber auch die Bücher, die unmittelbar nach der Krise erschienen sind, haben das Ende der Märkte zwar diskutiert, aber gleichzeitig nicht wirklich daran geglaubt («mitgedacht»). Ein Beispiel ist das Buch des weltberühmten Investors 
George Soros, Das Ende der Finanzmärkte - und deren Zukunft (Soros 2008). Das Buch von Nouriel Roubini heisst bezeichnenderweise ähnlich: Das Ende der Weltwirtschaft und ibre Zukunft (Roubini/Mihm 2011). Es wird entweder auf die selbstheilenden Kräfte des freien Marktes (wie die «unsichtbare Hand» von Adam Smith [1776/1978]; «Oikodizee» von Joseph Vogl [2012]; «Antifragilität» von Nassim Taleb [2012]) oder auf die erfolgreiche Einmischung des Staates (Keynesianismus; aktuell die Rettung des Finanzsystems durch die Zentralbanken während und nach der Krise 2008) rekurriert. Da man davon ausgeht, dass diese Kräfte nach wie vor wirksam sind, ist der radikale Zusammenbruch der Märkte nicht wirklich das Thema.

Allerdings - wenn man auf die Geschichte der Finanzmärkte zurückblickt - stellt man fest, dass es durchaus einige Präzedenzfälle gab, Situationen, in denen die Märkte im wahrsten Sinne des Wortes aufgehört haben zu existieren. So wurde in Russland 1917 und in China 1949 das private Vermögen enteignet; der Wertpapierhandel wurde reduziert und dann ganz verboten.

Wichtig ist es aber zu betonen, dass in beiden Fällen der Marktkollaps (oder das wortwörtliche Verschwinden der Märkte) mit einem radikalen Wechsel des politischen und wirtschaftlichen Systems zusammenfiel. Die Ursachen für beide «Apokalypsen» sind sozusagen nicht im System selbst zu suchen, sondern ausserhalb: Es waren externe, vor allem politische Kräfte.

Vor diesem Hintergrund stellt sich die Frage, ob die Entwicklung und Realisierung eines apokalyptischen Szenarios innerhalb des kapitalistischen Finanzsystems prinzipiell möglich wäre. Diese Diskussion wird heutzutage oft mit Bezug auf den algorithmischen Handel geführt; insbesondere wird das sogenannte High Frequency Trading (HFT) für gefährlich erachtet. Dabei handelt es sich um Computerprogramme, die sehr schnell - innerhalb von Bruchteilen einer Sekunde - die Marktsituation einschätzen und Geschäfte abschliessen. Nicht selten wird prophezeit, dass aus dem Zusammenspiel der Maschinen und Menschen oder sogar der sich komplett verselbstständigenden Algorithmen krisenhafte Regime entstehen könnten, die «non-renormalizable» («nicht mehr normalisierbar») sind (im Sinne von Sornette/Becke 2011). Sornette und von der Becke assoziieren die Risiken der HFT-Technologien eindeutig mit den systemischen Effekten dieser Technologien: Sie argumentieren, dass im High Frequency Trading Programme verwendet werden, die ähnlich auf die gleichen Informationen und Preisbewegungen reagieren und einander imitieren; hiermit verstärken sie die Herding-Effekte und «positive 
Feedbacks» $»^{1}$ im Markt. Dadurch könnten diese Programme den Markt so stark destabilisieren, dass ein Punkt in der Marktentwicklung erreicht wird, an dem das System kollabiert, weil es keinen Handel und keine Liquidität mehr gibt. Vogl (2012) nennt solche Ereignisse «Feedback-Katastrophen».

Als Beispiel wird oft der Flash Crash am 6. Mai 2010 diskutiert. An jenem Tag verlor der US Markt innerhalb von 20 Minuten fast eine Milliarde USD (9\% des Wertes). Einige Wertpapiere büssten zwischenzeitlich fast ihren gesamten Wert ein. Viele Marktteilnehmer (menschliche und elektronische) hatten aufgehört zu handeln, der Handel wurde unterbrochen, bis einige Käufer wieder in den Markt kamen und den Verkaufsdruck absorbierten. Es kam dann zu einer raschen Erholung.

Nach dem Flash Crash vom 6. Mai 2010 entfachte sich eine rege gesellschaftliche Diskussion über die Vorteile und Gefahren von HFT für die Märkte: Obwohl man nicht mit Sicherheit behaupten konnte, dass die HFTProgramme den Mini-Crash verursacht haben, wurde vermutet, dass sie ihn verstärkt und hiermit zur Destabilisierung der Märkte beigetragen haben. In diese Diskussion sind heutzutage verschiedene Marktbeteiligte, Interessenparteien und Kommentatoren involviert: die HFT-Firmen, Börsen, Handelsplattformen, Banken, Regulierer und Medien. Sie bringen ganz unterschiedliche Argumente ins Spiel, die von sehr unterschiedlichen Interessen getragen werden.

In einem Punkt sind sich aber alle einig: Die Natur der Märkte hat sich im letzten Jahrzehnt radikal verändert. Zum Beispiel analysierte das Foresight Project The Future of Computer Trading in Financial Markets, das vom Government Office of Science in UK durchgeführt wurde (Beddington u. a. 2012), die bestehenden und potenziellen Marktdynamiken, die der algorithmische Handel auslöst. Dieser Bericht bestätigte vor allem, dass die Bedeutung des algorithmischen Handels in den letzten Jahren stark gestiegen ist. So macht das High Frequency Trading heutzutage ungefähr $30 \%$ des gesamten Aktienhandelsvolumens in Grossbritannien und $60 \%$ in den USA aus. Nach anderen Angaben (Brogaard 2010, 11) beträgt der automatisierte Handel (inkl. HFT) über $77 \%$ des täglichen Aktienhandelsvolumens an den Börsen. Dadurch hat sich die Art und Weise, wie die Märkte funktionieren, prinzipiell verändert. Viele Entscheidungen, die das Handelsvolumen im Markt generieren, werden heutzutage anders getroffen als früher: Die automatisierten

1 «Positive feedback occurs when an action leads to consequences which themselves reinforce the action (Sornette/Woodard 2010, 22) 49 , am 26.04.2023, 11:31:34 
Kalkulationen spielen mindestens eine genauso grosse Rolle wie das menschliche Urteil. Die Geschwindigkeit, mit der die Algorithmen im Markt agieren, ist so hoch, dass ein Mensch das Geschehen kaum oder gar nicht in Echtzeit nachvollziehen - und daher nur mit Verzögerung reagieren - kann. Dieser Umstand gibt oft Anlass zur Behauptung, dass die algorithmischen Programme selbstständig handeln: Wenn sie eine profitable Handelsmöglichkeit entdecken, stellen sie die Orders in den Markt und schliessen Geschäfte ab; in diesem Sinne kann man sagen, dass sie Entscheidungen treffen. Es kommt auch oft vor, dass die Algorithmen miteinander handeln (oder dass sogar die Algorithmen ein und derselben HFT-Firma untereinander Geschäfte abschliessen - das sogenannte «self trading»). Hierbei haben wir es mit einem radikal neuen Regime zu tun. Märkte sind zu soziotechnischen Systemen geworden (Beddington u. a. 2012, 11), in denen Menschen und computerisierte Algorithmen gleichzeitig als Marktakteure auftreten. Persönliche Interaktionen werden teilweise durch «interaction orders of algorithms» (MacKenzie 2016) ersetzt; die Märkte werden zunehmend anonym (entpersönlicht).

Wir haben es also nicht mehr nur mit Menschen, die die (neuen, fortgeschrittenen) Maschinen und Technologien benutzen, zu tun, sondern auch mit Algorithmen, die - gleichzeitig mit den Menschen - Märkte beobachten, in den Märkten agieren und entscheiden. Natürlich verschwinden die menschlichen Akteure dadurch nicht; ihre Rolle in den Prozessen der Informationsverarbeitung und der Entscheidungsfindung verändert sich aber drastisch. Während einige von ihnen weiterhin als Händler alter Schule (mit Telefon und Bildschirm) agieren, fokussieren sich andere auf das Programmieren, Adjustieren und Überwachen von Algorithmen; wie genau die Aktivitäten von Programmierern und HFT-Betreibern den Markt beeinflussen, muss geklärt werden.

Allerdings muss man betonen, dass die Algorithmen in vieler Hinsicht anders als menschliche Trader handeln: «HFT can execute millions of times faster, but cannot use judgment to understand and interpret large and novel dislocations in real time» (Stafford 2015); sie verhandeln nicht und sind nicht imstande, die Marktnachrichten zu diskutieren, durch die Kommunikation eine gemeinsame Meinung zu bilden oder die Marktturbulenzen sinnvoll zu interpretieren. Gleichzeitig beeinflussen diese extrem schnellen algorithmischen «Autopiloten» im Wesentlichen, was heutzutage im Markt geschieht.

Die prominentere Rolle, die die Algorithmen jetzt spielen, erweckt oft die alten Ängste vor den Robotern, die ihre Erfinder vernichten («FrankensteinSyndrom»). Hier ist eine typische Stimme aus den Medien: 
«Allowing high-frequency trading as it is presently practiced will lead to a catastrophic market crash that will not only destroy trillions of dollars of wealth in America and around the world, it will destroy the economy in the process and ensure that a recovery won't be possible because the public won't have any faith in the capital markets that are supposed to be about capital formation, investment opportunity and risk transfer.» (Gilani 2012)

Es wird befürchtet, dass Bubbles und Crashs durch die Computerprogramme verursacht und verstärkt werden. Die Gegner von HFT prophezeien, dass irgendwann die sich häufenden Mini-Crashs ausser Kontrolle geraten und die HF-Händler die Märkte zerstören werden.

Obwohl die Spezialisten behaupten, dass diese «global-robotic-apocalypse» eigentlich noch nicht in Sicht ist und eher dem Bereich der ScienceFiction angehört (Future of Life Institute 2016), fokussiert sich die gesellschaftliche Debatte über HFT trotzdem sehr stark auf Bedrohungen für den Markt und erhält dadurch eine spezifische ethische Konnotation. Wie wir in Bezug auf die ganze Menschheit fragen können: Sind wir für den Erhalt des Lebens auf der Erde verantwortlich?, können wir in Bezug auf den Finanzmarkt fragen: Sind die Marktakteure für den Erhalt des Finanzmarktes verantwortlich?

In der Tat dreht sich die Diskussion über HFT vorwiegend um die Frage, was diese Art von Trading für die Märkte bedeutet. Dabei fokussieren sich die Parteien primär auf den Einfluss der computerisierten Handelsprogramme auf die Qualität der Märkte: ob der automatisierte Handel die Liquidität der Märkte erhöht oder senkt, ob er zu einer effizienteren Preisfindung beiträgt und die Transaktionskosten für alle Marktteilnehmer reduziert. Die Befürworter betonen, dass die schnellen Trader die verfügbaren Informationen augenblicklich verarbeiten und in die Marktpreise inkorporieren, dass sie die Effizienz und die Liquidität der Märkte erhöhen und die Bid-Ask Spreads (die Geld-Brief-Spanne) ${ }^{3}$ eng zu halten helfen. Die Gegner behaupten, dass

2 Vgl. Manfred Brockers Beitrag in diesem Band.

3 Der Bid-Ask Spread (die Geld-Brief-Spanne) ist die Differenz zwischen dem Bid-Preis und dem Ask-Preis eines Wertpapiers. Der Ask-Preis (auch Briefpreis genannt) ist der Preis, den der Verkäufer für das Wertpapier verlangt. Der Bidpreis (oder Geldpreis) ist der Preis, den der Käufer zu zahlen bereit ist. Es wird geglaubt, dass die Märkte umso effizienter sind, je enger die Geld-Brief-Spanne ist. Market-Makers sind Marktteilnehmer, die verbindliche Geld and Briefpreise stellen und hiernit den Markt liquide halten. 
HFT die Liquiditätsversorgung der Märkte eher unsicher macht, die Volatilität der Preise erhöht und - durch die Überflutung der Märkte mit Orders die Transaktionskosten aller Marktteilnehmer steigert.

Gleichzeitig wird nach der Finanzkrise erkannt, dass der veränderte Charakter der Finanzmärkte - das neue Marktregime - auch neue Arten von Risiken mit sich bringt, nämlich die Risiken der radikalen Marktinstabilität (der Marktzerstörung) durch die inneren (endogenen) Kräfte des Marktes. Diese Risiken werden als «systemische Risiken» diskutiert (Danielsson/Shin 2002; Danielsson u. a. 2009; Vogl 2012). Dabei handelt es sich um Risiken, die aus dem Zusammenspiel von individuellen Kalkülen und Handlungen der Marktakteure entstehen; dieses Zusammenspiel ist dem System nicht äusserlich, sondern immanent (endogen) und bedingt die unerwarteten und oft nicht intendierten Konsequenzen der individuellen Handlungen. So ein unerwartetes, nicht intendiertes Ereignis auf der Systemebene könnte eine gleichzeitige Pleite von mehreren Finanzinstituten sein, ein dramatischer Anstieg oder Absturz der Aktienpreise oder eben eine globale Finanzkrise: Es findet kein Handel mehr statt, weil es zum Beispiel keine Liquidität im Markt gibt oder weil die Marktteilnehmer einander nicht mehr vertrauen und keine Geschäfte abschliessen. Es geht dabei nicht um die Qualität der Märkte, sondern um den Erhalt oder die Zerstörung der Märkte.

Dass die Finanzmärkte solche destabilisierenden Kräfte permanent entwickeln, ist nicht neu. Schon 1970 hat Hyman Minsky eine «financial instability hypothesis» aufgestellt und behauptet, dass rationales Verhalten der Akteure auf der Mikroebene auf keinen Fall ein Gleichgewicht und einen krisenfreien Ablauf auf der Makroebene garantiert. Alle Krisen sind Folgen der Eigendynamik der Märkte: «Der Finanzmarkt [wird] durch seine Ruhe beunruhigt, durch seine Stabilität destabilisiert, und gerade sein effizientes Funktionieren wird ganz und gar dysfunktional.» (Vogl 2012, 162)

Danielsson und Shin (2002) benutzen die Ereignisse während der Eröffnung der Millennium Bridge in London im Jahr 2000, um die Idee der systemischen Risiken zu illustrieren. Die Brücke geriet in eine leichte Bewegung (durch einen Windstoss?) und mehrere Menschen auf der Brücke fingen an, synchron nach rechts und links zu treten, um ihr Gleichgewicht zu halten. Die Schwingungen der Brücke wurden stärker, woraufhin die Fussgänger ihre Ausgleichsbewegungen noch stärker synchronisierten: Die Brücke wackelte dadurch schliesslich so stark, dass die Situation zu gefährlich wurde und die Brücke geschlossen werden musste.

Die Metapher der Millennium-Brücke für die systemischen Risiken illustriert die wichtigsten Charakteristiken dieser Risiken. Sie sind nicht von einer 
einzelnen Person oder einem Kollektiv der Akteure intendiert und herbeigeführt; eher wird das Verhalten der einzelnen Marktbeteiligten ungewollt koordiniert, sodass es zu Trendverstärkung und Herding kommt. Das Verhalten auf der Mikroebene wird zu den (unerwünschten) Effekten und Ereignissen auf der Makroebene aggregiert. Die zentrale Frage in diesem Zusammenhang lautet: Können die Individuen (Mikroebene) für die kollektiven Outcomes (Makroebene), die sie nicht intendieren, verantwortlich gemacht werden? Und: Ist diese Frage moralisch relevant?

Die eindeutige Antwort in der Literatur ist, dass es sich hierbei in der Tat um wichtige ethische Probleme handelt. Die unerwünschten Effekte betreffen nicht nur diejenigen, die unmittelbar im Markt agieren. Die Verursacher systemischer Risiken sind nicht unbedingt die Betroffenen; Risiken werden auf andere übertragen, sodass sich eine «soziale Risikosituation» ergibt (Heinemann 2011, 6) oder eben Gefahren (Vogl 2012, 177):

«Weil es zur Eigenart kapitalistischer Ökonomie gehört, dass die Folgen ihrer riskanten Entscheidungsprozesse auch diejenigen zu spüren bekommen, die nicht an den Entscheidungen teilhaben, und weil sich Risiken von Gefahren dadurch unterscheiden, dass sich letztere nicht dem eigenen Tun oder Unterlassen zurechnen lassen, haben sich ökonomische Systemrisiken und kalkulierbare Schadensfälle für die Mehrzahl derjenigen, die in aller Abhängigkeit nichts zu entscheiden haben, in elementare Gefahren verwandelt. Hier überschreiten die Risiken, auf die man sich im normalen Funktionsablauf eingelassen hat, die Möglichkeit rationaler Kalkulation, und wahrscheinlich muss man sich nicht darüber wundern, dass so operierende Gesellschaften «Angst vor sich selbst bekommen.»

Aus diesem Grund kommt systemischen Risiken eine besondere moralische Bedeutung zu. Heinemann $(2014,228)$ behauptet, dass systemische Risiken im Finanzmarkt «moralisch inakzeptable Risiken» sind, die durch menschliches Handeln hervorgebracht werden; daher solle man Risikoethik in Bezug auf diese Art von Risiken als Verantwortungsethik verstehen:

«Das heisst, da ein moralisch relevanter Schaden erst eintritt, wenn Akteure zuvor ihre Verantwortung vernachlässigt haben bzw. wenn sie ex ante keine Massnahmen zu dessen Verhinderung getroffen haben, muss und kann ex ante ein aktiver verantwortungsvoller Umgang mit systemischen Risiken gefordert werden, der den Ausbruch einer Krise verhindert.»

Diese radikale Aussage, die eine vollständige Kontrollierbarkeit solcher Ereignisse wie Finanzmarktkrisen impliziert, möchte ich in meinem Beitrag hinterfragen. In dem Zitat oben wird - im Sinne der «Oikodizee» (Vogl 2012) - 
vorausgesetzt, dass es möglich ist, eindeutig zu identifizieren, wer der Verursacher systemischer Risiken ist (moral agency), sodass man die Verantwortung für die Schäden, die eventuell oder tatsächlich eintreten, eindeutig zuschreiben kann. Bei näherem Hinsehen scheint die Beantwortung dieser Fragen nach Risikozumutung und Verantwortungszuschreibung - insbesondere im Fall eines hoch technologisierten Finanzmarkts - nicht trivial zu sein.

Es stellt sich nämlich die Frage, ob eine klassische, verantwortungsbasierte Ethik in diesen Zusammenhängen eigentlich ein angemessenes Analyseinstrument ist. Denn deren Voraussetzung, einzelne oder kollektive Subjekte, denen Handlungen und deren Konsequenzen klar zugeschrieben werden können, hat gerade durch die Veränderungsprozesse im Markt möglicherweise gar keinen Platz. Dort, wo eine Verschiebung der entscheidenden Prozesse weg von den menschlichen Akteuren hin zu maschinellen stattfindet, wo Maschinen «Entscheidungen treffen», müsste entweder eine ganz andere Art der Ethik Anwendung finden - oder dem Humanum mehr Raum eingeräumt werden. $\mathrm{Ob}$ allerdings eine dieser beiden Möglichkeiten erfolgversprechend ist, kann abschliessend nicht klar gesagt werden.

In meinem Beitrag folge ich dem Ansatz von Coeckelbergh (2012), der die Verantwortung für das technologische Handeln an zwei aristotelische Voraussetzungen aus der Nikomachischen Ethik knüpft: Erstens müssen die Handelnden genau verstehen, was sie tun, das heisst, um die potenziellen Folgen ihrer Handlungen wissen, sowie zweitens diese Folgen kontrollieren können. Laut Coeckelbergh (2012) sind diese Bedingungen im Fall eines Handelns, bei dem Technologie eine grosse Rolle spielt, nicht erfüllt. Im Folgenden entwickle und illustriere ich diese Behauptung, indem ich offizielle Berichte über zwei Mini-Crashs (am 6. Mai 2010 und 15. Oktober 2014) analysiere. Meine Analyse demonstriert, dass weder die Wissensbedingung noch die Kontrollbedingung in den modernen «hybriden» Finanzmärkten, wo Menschen und Maschinen koagieren, erfüllt ist, sodass die Suche nach den Verantwortlichen sich als extrem schwierig gestaltet. Eine rigorose Vorgehensweise (wie im Zitat von Heinemann oben) - sie wissen, was sie tun, und sollen ibr Handeln so gestalten, dass es keine systemischen Risiken herbeiführt - funktioniert einfach nicht. Es bleibt unklar, wer die relevanten Marktakteure überhaupt sind. Können in einem Marktregime, in dem Algorithmen auch Entscheidungen treffen, diese technischen Objekte für das Geschehen im Markt (mit)verantwortlich gemacht werden? Wer oder was genau ist «moral agency»? Weiterhin: Wie können (menschliche und nichtmenschliche) Marktakteure systemische Risiken, die sie nicht intendieren, nicht genau verstehen und nicht kontrollieren, verantworten? Wir haben es hier eindeutig mit der Diskrepanz zwischen dem 
normativen Charakter jeder Ethik und der Unterbestimmtheit menschlichen Handelns zu tun.

Diese Diskrepanz wird am besten im Konzept der Tragödie (oder eher Tragik) von Kierkegaard erfasst, auf das Coeckelbergh (2012) in seiner Analyse der Verantwortung für technological acts rekurriert. Mit diesem Konzept weist er auf die Grenzen der Kontrollierbarkeit der Technik und die Beschränktheit des Wissens über die relevanten Kausalmechanismen und Folgen der Technikanwendung hin. Mit Kierkegaard plädiert er im Anschluss für eine Ethik, die weniger rigoros ist und keine radikale Verantwortungszuschreibung verlangt. Ich werde im Folgenden seine Analyse auf die Fälle des High Frequency Trading und der damit verbundenen systemischen Risiken anwenden.

Damit sollen diese Überlegungen zur Schliessung einiger wesentlicher Lücken in der bestehenden Literatur beitragen. Erstens hat die Ethik bis jetzt das Feld des automatisierten Handels nicht eingehend bearbeitet. Dies ist nicht verwunderlich, wenn man bedenkt, dass eine Ethik des Finanzmarktes generell so gut wie nicht existent ist (Boatright 2010). Das Thema HFT und Ethik wird in sehr wenigen Beiträgen behandelt, etwa bei Angel und McCabe (2013), Davis u. a. (2013), Coeckelbergh (2015) und Cooper u. a. (2016). Zweitens wird der ethische Umgang mit systemischen Risiken in hoch technologisierten Finanzmärkten ebenfalls kaum behandelt. In den gerade erwähnten Publikationen über HFT und Ethik werden unter «Ethik» ganz unterschiedliche Aspekte diskutiert wie zum Beispiel Fairness und professionelle Verhaltenskodizes für die Marktakteure. Die Fragen nach der Verantwortung für den Erhalt der Märkte, systemische Risiken und nach ethischen Aspekten des Zusammenspiels zwischen Mensch und Maschine in den modernen Finanzmärkten werden weitgehend ausser Acht gelassen.

Zu zeigen, dass die Frage nach Technologie und Verantwortung nicht nur für Ingenieure und Programmierer relevant ist, ist für das Verstehen der Debatte, die in der Gesellschaft rund um HFT und Finanzmarkt geführt wird, wichtig. Es wird ein richtiger Kampf um «moral orders» (Fourcade/Healy 2007) der automatisierten Märkte ausgetragen, in den mehrere Parteien involviert sind: private und institutionelle Anleger, die HFT-Firmen, Börsen, Anwälte, Wissenschaftler, Blogger und Regulierer. Jede Partei hat ihre eigene Vorstellung über die moralischen Werte der Märkte und ihrer Beteiligten und verteidigt sie vehement. In diesem Kampf um «moral orders» werden normative Bedeutungen und moralische Werte geformt und verändert (Fourcade/Healy 2007, 301). Dabei werden moralische Kategorien eher impliziert als explizit diskutiert. Dies führt dazu, dass die Streitenden in argumentative 
Sackgassen geraten, aneinander vorbeireden und über wechselseitige Beschuldigungen nicht hinauskommen. Es ist deswegen interessant und wichtig, diese gesellschaftlichen Debatten mit der reflexiven Ebene der Moralphilosophie zu verbinden. Durch diesen Schritt können die argumentativen Schwierigkeiten und Sackgassen explizit gemacht werden, sodass Wege zu ihrer Überwindung aufgezeigt werden können.

\section{Algorithmischer Handel: Was ist das?}

Der algorithmische Handel basiert auf Computerprogrammen, die Kaufund Verkaufsorders automatisch in einem elektronischen Handelssystem generieren. Den Kern des algorithmischen Handels bildet das Orderbuch, eine Maschine, in der die Orders gesammelt und gematcht werden. Die Preise und der Umfang des Geschäfts werden automatisch, ohne die früher üblichen Verhandlungen zwischen den menschlichen Kontrahenten, ermittelt. Die Programme handeln meist tagsüber, stellen aber ihre Positionen glatt über die Nacht, das heisst, sie neutralisieren die von ihnen tagsüber eingegangenen Risiken. Das High Frequency Trading wird von spezialisierten Firmen betrieben, aber auch von Banken, die eine besondere Abteilung dafür haben (the broker-dealer proprietary desk), und von den Hedge-Funds.

Dabei ist es irreführend, über HFT generell zu sprechen. Die HFTFirmen verfolgen ganz unterschiedliche Strategien, oft gleichzeitig. Einige von ihnen spezialisieren sich auf den Handel: Sie verkleinern die Orders, die an die Orderbücher übermittelt werden, sodass viele kleine Positionen gleichzeitig auf unterschiedlichen Börsen angenommen werden. Dadurch werden grosse Preisbewegungen, die normalerweise durch grosse Orders ausgelöst werden, vermieden. Die meisten HFT-Firmen agieren wie Market-Makers, das heisst, sie stellen permanent die Bid- und Ask-Preise und unterstützen die Liquidität im Markt.

Eine andere Kategorie der Algorithmen wurde mit dem Ziel entwickelt, nicht nur zu handeln, sondern auch Profite zu erzielen. Einige algorithmische Handelsprogramme beobachten den Markt und nutzen minimale Preisdifferenzen zwischen den Handelsplätzen (Börsen) und Wertpapieren aus, um profitable Geschäfte abzuschliessen (mit anderen Worten: sie betreiben Arbitrage). Da die Arbitragegelegenheiten in den heutigen Märkten sehr selten oder sehr gering sind, fokussieren sich die algorithmischen (und vor allem die HFT-)Programme auf unterschiedliche Techniken, die kurzfristige Preisprognosen machen und ausnutzen. MacKenzie $(2016,15)$ beschreibt, zum 
Beispiel, die order-book dynamics: Diese einfache Strategie stellt fest, ob das Angebot an einem bestimmten Wertpapier höher ist als die Nachfrage oder umgekehrt; wenn das Angebot höher ist als die Nachfrage, kann man mit hoher Wahrscheinlichkeit erwarten, dass die nächste Preisbewegung nach unten geht. Die kurzfristigen Preisvorhersagen können auch auf der Basis der Marktund Unternehmensnachrichten oder Preisbewegungen ähnlicher Wertpapiere erfolgen.

Nicht nur die erfolgreiche Informationsverarbeitung (wie in den traditionellen Märkten) spielt eine Rolle im algorithmischen Handel, sondern auch die räumliche Nähe eines Computers zum Börsenserver. Dies erlaubt, die notwendigen Milli-/Nanosekunden zu gewinnen, um schneller als der Markt zu sein. Strategien, die auf latency ${ }^{4}$ reduction und co-location basieren, sind zum Beispiel flash trading und latency arbitrage. Flash Trading bedeutet, dass diejenigen, die ihre Computer an der Börse besonders günstig (nahe am Server) platziert haben, die Informationen über Preise oder Orders ein paar Millisekunden schneller bekommen als die anderen Marktteilnehmer (eine Millisekunde beträgt 0,01 Sekunden). Die High Frequency Traders bekommen diese Informationen, bevor die Daten an den offiziellen Handelsplattformen publiziert werden. In diesem Zusammenhang wurden öfter ethische Bedenken erhoben, dass eine «Zweiklassengesellschaft» an den Börsen entstehe. Der Grundsatz der Märkte - gleicher Zugang zu den Informationen für alle Marktteilnehmer - werde dadurch verletzt. Wir kommen später zu diesem Punkt. Latency arbitrage ist eine Strategie, die auf reinem Geschwindigkeitsvorteil basiert: Man handelt zwischen den Börsen und versucht dabei, sich schneller als die anderen in den Schlangen für die Orders zu platzieren.

Latency reduction und co-location eröffnen Wege für die weiteren sogenannten «gerichteten» HFT-Strategien. Zum Beispiel gibt es Algorithmen, die das Momentum (einen bestimmten Preistrend) im Markt initiieren, um im nächsten Schritt selber von diesem Trend zu profitieren. So können die Handelsprogramme Orders generieren, die oft storniert und nicht ausgeführt werden. Dadurch wird eine Nachfrage (ein Angebot) vorgetäuscht und Käufe (Verkäufe) initiiert, die einen Preis in eine bestimmte Richtung lenken. McNamara $(2016,114)$ liefert ein Beispiel für diese sogenannte Spoofing-Strategie: 
«A simple example of this might be if the $\mathrm{NBBO}^{5}$ for General Motors stock consists of a bid at $\$ 15.10$ and an offer at $\$ 15.14$. «Spoofing) would involve making an offer to buy (a bid) at $\$ 15.12$, then executing the opposite transaction, selling the security at this price, after other players in the market have raised their bids in response to the higher offer. Finally, the original offer to buy at $\$ 15.12$ will be cancelled before other parties can act on it. In this way prices in the market are manipulated higher or lower without the manipulating party having to act on the quotes it has placed on an exchange or other trading venue. The fact that an estimated $95 \%$ of orders entered onto markets are cancelled may illustrate how frequently this technique, along with quote-stuffing, is used.»

Die HFT-Programme, die quote stuffing betreiben, schicken sehr viele Orders in den Markt und löschen sie ebenso schnell, um den Markt zu testen, aber auch die Programme der Konkurrenten zu irritieren oder zu verlangsamen. Die fremden Algorithmen können die «fiktiven» Anfragen von den «echten» nicht unterscheiden, reagieren auf sie, als ob sie normale Orders wären, und verfolgen den dadurch initiierten Preispfad. Der Preis bewegt sich hiermit in die für die HFT-Strategie günstige Richtung.

Mit der ansteigenden Bedeutung der HFT-Programme für das Geschehen im Markt hat sich die Natur der Finanzmärkte folglich verändert: Es geht nicht darum, dass die menschlichen Akteure komplett verschwinden oder irrelevant werden, sondern dass die Beziehung zwischen Händlern und Märkten jetzt durch Algorithmen «vermittelt» («mediated») wird (auch Lenglet 2011; 2013). Die unmittelbare Mensch-zu-Mensch-Interaktion auf dem Börsenparkett und das spätere Mensch-zu-Screen-Zusammenspiel (wo die Preise auf den Bildschirmen den Markt repräsentieren und die Trader per Telefon und Chat über diesen auf dem Screen abgebildeten Markt kommunizieren; Knorr-Cetina/Brügger 2002) wurden teilweise durch die Trader ersetzt, die mithilfe von Algorithmen im Markt agieren oder durch die Algorithmen repräsentiert werden.

Deswegen könnte man sagen, dass wir es auf der Oberfläche mit einer anonymen (entpersönlichten) Form des Marktes zu tun haben; in der Tiefe aber sind die menschlichen Akteure nach wie vor sehr wichtig, wenn auch nicht entscheidend (ihre Rolle wird noch ausführlicher beschrieben). Und es ist nicht zu vergessen, dass alle Trades, die nicht mit Beteiligung der Algorithmen ab-

5 NBBO bedeutet National Best Bid and Offer: Das sind die Brief- und Geldkurse, die ein durchschnittlicher/Anleger normalerwerse sieht.49, am 26.04.2023, 11:31:34 
geschlossen werden, von den menschlichen Händlern - mit Screen und Telefon - getätigt werden, und das sind nach wie vor nicht vernachlässigbare 40$60 \%$. Es geht daher um eine einmalige Symbiose von Maschinen und Menschen an einem virtuellen Ort - dem Markt. Der neue, vollständig maschinengesteuerte Zustand ist bei weitem noch nicht erreicht.

Gleichzeitig ist noch einmal zu betonen, dass die Algorithmen in vieler Hinsicht anders als menschliche Trader agieren: Sie bilden keine qualitativen Urteile, verhandeln nicht und sind nicht imstande, die Marktnachrichten zu diskutieren oder durch Kommunikation eine gemeinsame Meinung zu bilden. Die menschlichen Akteure tun aber genau dies - sie kommunizieren, interpretieren und lassen sich von Emotionen treiben. Deswegen haben wir einen Markt, in dem die «algorithmischen Autopiloten» mit Menschen koexistieren, ein soziotechnisches System eben, wie wir es heutzutage häufig in der Medizin oder im Luftverkehr beobachten. Dabei hat der erhöhte Einsatz der komplexen und teilweise autonomen Technologien in der Medizin zum Beispiel die ethischen Debatten stark geprägt. Genau dies soll auch in Bezug auf die modernen «soziotechnischen» Finanzmärkte geschehen.

\section{Ethische Probleme von HFT: Vorüberlegungen}

Was können wir in einem Markt, wo Algorithmen und Menschen koagieren, aus der Perspektive der Ethik und Moral erwarten? Einerseits wirft der kurze Überblick der HFT-Strategien in dem vorherigen Abschnitt viele ethische Fragen auf. Es ist offensichtlich, dass «[a]utomation has changed those whom trading can harm, how they might be harmed, and the scale of the harm» (Davis u. a. 2013, 852). Andererseits werden genuin ethische Überlegungen über die HFT selten und nur vereinzelt angestellt. Sie drehen sich dann primär um drei Themen: den Marktmissbrauch, die Marktqualität und die Marktstabilität.

Die Probleme, die mit dem Marktmissbrauch zusammenhängen, haben die offensichtlichsten ethischen Implikationen, zum Beispiel die Fragen der Fairness (Angel/McGabe 2013). Es wird diskutiert, ob die HFT-Strategien eine Zwei-Klassen-Gesellschaft im Markt kreieren, in der die High-FrequencyHändler technologisch überlegen sind und dadurch einen Vorteil in der Geschwindigkeit dank co-location sowie einen bevorzugten Zugang zu den wichtigen Informationen wie im Falle von flash orders geniessen. Zum Beispiel erhalten die HFT-Firmen die Informationen über die Orders schneller als der Rest des Marktes, stellen sich ganz vorne in der Schlange für die Ausführung 
bestimmter Orders und machen Profite auf Kosten von anderen, so der Vorwurf. Dies widerspricht der Idee effizienter Märkte.

Weiterhin wird argumentiert, dass die HFT-Praktiken eine Marktmanipulation und somit einen Marktmissbrauch darstellen: Die Preise werden kurzfristig in eine bestimmte Richtung gepusht, wobei die Pusher davon profitieren. Ausserdem führen einige HFT-Strategien wie spoofing und quote stuffing dazu, dass ein Investor, der auf einen von HFT gesendeten Bid oder Ask reagiert, die Transaktion zu dem auf dem Screen angezeigten Preis/Limit oft nicht durchführen kann; er hat dann mit einem «Phantommarkt» zu tun: Dieses Phänomen wurde in Michael Lewis' Buch The Flash Boys beschrieben. Das bedeutet, dass implizite Transaktionskosten für die Anleger steigen und dass der Markt für sie nicht richtig funktioniert. Das Versenden von «fiktiven» Bids oder Asks hat mit dem Versenden von Spam-E-Mails eine Gemeinsamkeit: Es ist für den Sender zwar praktisch kostenlos, für den Empfänger jedoch nicht (Nagel/Zajonz 2012).

Hier werde ich mich mit dem Thema Marktmissbrauch und Marktmanipulation allerdings nicht direkt beschäftigen und eher auf die Problematik fokussieren, die um kollektive Outcomes und systemische Risiken kreist: die Marktqualität und die Marktstabilität. Dieser Fokus ist deshalb berechtigt, weil die Hauptdiskussion bezüglich HFT in der Finanzindustrie (aber auch in der Presse) sich eben primär um die Bedeutung des algorithmischen Handels für die Marktqualität und die Marktstabilität dreht. Initiiert wurde diese Diskussion durch die zahlreichen Mini-Crashs, die immer häufiger die modernen Finanzmärkte plagen. Die Mini-Crashs sind Marktentwicklungen, während derer die Titel ohne fundamentale Begründung innerhalb von wenigen Sekunden sehr stark an Wert verlieren und sich dann rasant erholen. Es ist unumstritten, dass solche Crashs (wie am 6. Mai 2010) sich häufen. Es wird auch gesehen, dass sie - obwohl es zu einer schnellen Erholung der Märkte kommt - nicht ganz harmlos sind. Sie verursachen zusätzliche Kosten für die Investoren, auch für diejenigen, die kein HFT betreiben. Zum Beispiel sind, wenn Privatanleger Orders mit stop-loss platzieren (einer Kursmarke, bei deren Unterschreitung die Wertpapiere automatisch verkauft werden) und der Kurs nur für wenige Sekunden einbricht, die Papiere zu einem niedrigen Preis aus dem Depot verkauft. Ob der Kurs sich in ein paar Sekunden wieder erholt, spielt keine Rolle; wenn man das Wertpapier aus fundamentalen Gründen doch im Depot haben möchte, muss man es wieder - teurer - kaufen.

Gleichzeitig wird die Diskussion darüber geführt, dass die HFTStrategien nicht nur den einzelnen Anlegern schaden oder ihnen gegenüber 
unfair sind, sondern den Markt insgesamt destabilisieren. Es wird heftig darüber gestritten, ob die HFT-Programme die besagten Mini-Crashs verursachen sowie grundsätzlich die Liquidität am Markt «absaugen» und die Volatilität sowie die Kosten für Anleger erhöhen. Eine einheitliche Meinung gibt es dazu nicht (Menkveld [2016] und Beddington u. a. [2012] liefern einen hervorragenden Überblick über diese Debatte).

Während einige Studien behaupten, dass die HFT-Programme die Liquidität der Märkte verschlechtern, insbesondere in turbulenten Zeiten, finden andere keine Evidenz dafür (vgl. Brogaard 2010). Ähnlich steht es um die Qualität der Preisfindung: Einige Untersuchungen (z. B. Carrion 2013) zeigen, dass die neuen Informationen an den Tagen, an denen die Beteiligung der algorithmischen Programme hoch war, viel schneller in die Preise inkorporiert wurden, was eine Erhöhung der Effizienz der Märkte suggeriert. Brogaard u. a. (2014) behaupten auch, dass der Beitrag der HFT-Algorithmen zu einer effizienten Preisfindung positiv ist, vor allem, weil sie aktiv Preis-Umkehr-Strategien betreiben und hiermit effiziente Preise «suchen». Auch die aktive (statistische) Arbitrage als eine der populärsten HFT-Strategien komme der Preiseffizienz der Märkte zugute. Allerdings weisen andere Untersuchungen darauf hin, dass ähnliche Reaktionen der Algorithmen auf die News zu Preisübertreibungen und starken Abweichungen von fundamentalen Daten führen können (Benos/Sagade 2012). Generell behauptet Weller (2016), dass die HFT-Beteiligung den Informationsgehalt der Preise reduziert und zu Informationsverlusten führt.

Gleichzeitig wird auf die weiteren destabilisierenden Effekte von HFT hingewiesen. Es wird behauptet, dass die Handelssysteme häufig von der Flut der fiktiven Orders überlastet werden: Einzelne HFT-Algorithmen generieren für einige Wertpapiere mehrere Zehntausend Quotes pro Sekunde.

«Der gegenwärtige Rekord an Quotes pro Sekunde, der von einem einzigen HFT-Akteur an einer einzelnen Börse aufgestellt wurde (Stand 24. April 2012), wird von einer US-Aktie gehalten (nämlich PSS World Medical) und liegt bei 47 138. Zum Vergleich: in einer Sekunde verarbeitet Google derzeit ca. 34000 Suchanfragen weltweit, es gibt kaum mehr als 10000 Twitter 'Tweets` und es fallen bei Facebook maximal etwa 6000 Status Updates von Profilen an.» (Nagel 2012)

Die Datenflut ist enorm, allerdings gibt es keine klaren Hinweise darauf, dass sie den Handel nachhaltig gefährdet.

Weiterhin werden die Märkte angeblich nicht nur durch diese Flut der (oft fiktiven) Orders destabilisiert, sondern auch durch die verkürzte Halte- 
dauer der Wertpapiere. Durch den aktiven Einsatz von HFT geht diese Haltedauer kontinuierlich zurück, nämlich von ein paar Jahren noch vor kurzem auf durchschnittlich 10 Sekunden heutzutage. Zum Vergleich: Für USAktien betrug die durchschnittliche Haltedauer am Ende des Zweiten Weltkriegs 4 Jahre, im Jahr 20008 Monate, 20082 Monate und 2011 nur noch 22 Sekunden (Patterson 2012); diese Zahlen werden kontrovers diskutiert (Turbeville 2013), doch die Tendenz ist eindeutig. Der häufige Handel trägt zur Volatilität der Märkte bei. Ausserdem führen die Verluste, die Anleger aufgrund von fiktiven Orders oder Stop-Loss-Ausführungen erleiden, dazu, dass ihr Vertrauen in den Markt geschwächt wird; dies wirkt ebenso destabilisierend auf die Märkte.

Diese und andere Argumente werden in den Debatten über HFT, die in der Presse, in den Gerichtssälen, in Internetblogs und in akademischen Zeitschriften geführt werden, immer wieder vorgebracht. Dabei geht es sehr oft um die Suche nach den Verantwortlichen für unerwünschte Marktentwicklungen. Es wird vorausgesetzt, dass, wenn es gelingt, eine klare kausale Kette zwischen den Aktivitäten der HF-Händler und der Marktstabilität aufzuweisen, die Frage nach der Verantwortung eindeutig gelöst werden wird. Das gelingt aber nicht so direkt. Das möchte ich zeigen, indem ich die offiziellen Reports über zwei Mini-Crashs analysiere, nämlich den Bericht der Commodity Futures Trading Commission (CFTC) und der Securities and Exchange Commission (SEC), Findings Regarding the Market Events of May 6, 2010 (CFTS/SEC 2010), sowie den Report der US Treasury Commission, The US Treasury Market on October 15, 2014 (Treasury 2015). In beiden Berichten wird die Rolle von HFT für die Entstehung und den Verlauf der Mini-Crashs eingehend untersucht, um letztendlich die Frage zu beantworten, ob das HFT für beide Crashs verantwortlich gemacht werden kann. Bemerkenswert ist, dass keine eindeutige Antwort auf diese Frage gegeben wird.

\section{Zwei Mini-Crashs: Was lernen wir daraus?}

Das zentrale und unumstrittene Ziel - und das moralische Ideal - der Finanzmarktregulierer wurde im Consultation Report des technischen Komitees von der International Organization of Securities Commissions (OICUIOSCO 2011, 9) formuliert:

«Financial markets should be efficient, fair, orderly and transparent. Investors should easily and rapidly be able to determine the best available price in the market. Access to markets should be fair. Deep and liquid markets create opportunities for listed companies toraise funds and opportunities for participants to invest 
and to manage risk. They also embody active and efficient price formation through trading, quickly pricing in news and reflecting changing attitude towards risk.»

Diesem höchsten moralischen Ideal sollen alle Marktteilnehmer, inklusive HFT, dienen; ihr moralischer Wert wird aus dieser Perspektive eingeschätzt und im Sinne der Nützlichkeit für die Markteffizienz und Marktintegrität verstanden. Unter diesem Blickwinkel wurden beide Ereignisse - der Flash Crash am 6. Mai 2010 und der US-Treasury-Mini-Crash (oder eher Mini-Rallye, d. h. ein starker Preisanstieg) am 15. Oktober 2014 - in den Berichten der Regulierer analysiert.

Am 6. Mai 2010 hatte eine grosse Verkaufsorder in den ohnehin nervösen und volatilen Märkten einen starken, wiewohl kurzfristigen Preiseinbruch ausgelöst. Diese Order wurde von einem Trader (einem Menschen) initiiert, aber von einem einfachen Handelsalgorithmus ausgeführt. Der Trader hatte den Verkauf nicht hinsichtlich Preis und Zeitspanne limitiert, sodass der Algorithmus einfach «blind» verkaufte, auch wenn die Preise schon abzustürzen begannen.

Dies löste eine erschreckende Preisdynamik aus. Die Untersuchung der SEC, die fünf Monate später publiziert wurde, schilderte die HFTProgramme als wichtige Beitragende zu den dramatischen Marktentwicklungen und löste eine gesellschaftliche Debatte über sie aus. In dem Report wurde berichtet, dass die HFT-Firmen durch den sogenannten «Hot potato»Effekt den Preistrend nach unten verstärkt hätten: Sie hätten begonnen, schnell und aggressiv - parallel zu der ursprünglichen grossen Verkaufsorder im Markt - zu verkaufen, um dann sofort wieder zu kaufen: Sie haben die Wertpapiere wie heisse Kartoffeln herumgereicht. Dabei war der HFT-Anteil im Handelsvolumen an jenem Tag bedeutend: «Between 2:45:13 and 2:45:27, HFTs traded over 27000 contracts, which accounted for about 49 percent of the total trading volume.» (Sornette/Becke 2011,11) Als allerdings die Preise im Futures-Markt und dann im Aktienmarkt (wegen des Spillover-Effekts) schnell zu fallen begannen, haben einige HFT-Programme (aber auch menschliche Händler) «Handelspausen» eingelegt; andere haben aber weiter verkauft. Dieser Entzug der Liquidität verschlimmerte den Preissturz, weil fast niemand im Markt war, um den Verkaufsdruck zu absorbieren. Der SEC-Bericht betonte hiermit, dass, auch wenn die HFT-Firmen die Bereitstellung der Liquidität im Markt generell unterstützen, die Qualität dieser Liquidität fraglich ist: Sie kann durch die algorithmischen Programme sehr schnell entzogen werden und eine kleine oder grosse Krise verstärken; mit anderen Worten, die Liquidität wird durch HFT volatil und unsicher. Auch 
die akademische Forschung, zum Beispiel Kirilenko u. a. (2016) sowie Sornette/Becke (2011), argumentiert, dass die HFT-Programme erheblich zu dem Mini-Crash am 6. Mai 2010 beigetragen haben. Jedoch können diese Programme nicht als alleinige Verursacher betrachtet werden: Der Report der SEC tendiert eher dazu, die unlimitierten Verkaufsorders, mit denen ein Trader in den Markt ging, für den Auslöser des Mini-Crashs und damit für hauptverantwortlich zu halten.

Allerdings hat die Suche nach den «Sündenböcken» für den Mini-Crash am 6. Mai 2010 viel später, nämlich 2015, eine interessante Wende genommen. Ein einzelner HF-Trader (Navinder Singh Sarao), der privat in seinem Haus in einem Londoner Vorort handelte, wurde der absichtlichen Marktmanipulation an dem Crash-Tag beschuldigt (Scannell/Stafford 2015). Er wurde verhaftet und musste vor Gericht die Verursachung des Mini-Crashs am 6. Mai 2010 verantworten. Er hat angeblich die Spoofing-Strategie benutzt (schnelle Platzierung und Stornierung mehrerer Orders, um von dem ausgelösten Preistrend zu profitieren). Diese Entwicklung in der Suche nach einem Schuldigen ist in vieler Hinsicht bemerkenswert: Erstens, weil in einem zum grössten Teil automatisierten Markt doch ein Mensch für die ungünstigen Entwicklungen verantwortlich gemacht wird. Zweitens:

«[T]he unmasking of Mr Sarao has raised eyebrows among traders and the broader industry. Markets by their nature reflect the choices and actions of numerous people and no single trade or activity can be held responsible for big market swings.» (Scannell/Stafford 2015)

Wir werden später auf diesen Punkt zu sprechen kommen.

Die Kontroverse um den Mini-Crash von 2010 demonstriert, dass keine klaren Aussagen über die Ursachen und Verantwortlichen gemacht werden können: $\mathrm{Ob}$ dafür einzelne Händler oder die HFT-Firmen verantwortlich sind, ist unmöglich zu sagen. Dies bestätigt auch der US-Treasury-Bericht über den Mini-Crash am 15. Oktober 2014.

An jenem Tag erlebte der US-Treasury-Markt eine extrem hohe Volatilität: Die Preise stiegen plötzlich (und die Renditen fielen dementsprechend stark: auf 1,9\%, am Tag davor hatte der Markt mit 2,2\% geschlossen; das ist für den US-Treasury-Markt eine ausserordentlich starke Bewegung). Die Liquidität war aus dem Markt verschwunden, was bei einem so zuverlässigen und häufig gehandelten Wertpapier wie der 10-jährigen US-Treasury-Anleihe äusserst ungewöhnlich, eigentlich unmöglich ist. Der Markt erholte sich aber vollständig - und sehr schnell. Die ganze Episode spielte sich innerhalb von 
12 Minuten ab: zwischen 9.33 und 9.45 Uhr. Obwohl das gesamte Marktsentiment an dem Tag etwas negativ war, gab es keine Nachrichten, die eine derart starke Marktbewegung gerechtfertigt hätten.

Nach dem Mini-Crash wurde eine US-Treasury-Kommission beauftragt, dieses besorgniserregende Ereignis zu untersuchen. Die Kommission stellte fest, dass an jenem Tag zwei Gruppen von Marktteilnehmern besonders aktiv wurden: Einerseits waren die proprietary trading firms (PTF, ein anderer Name für HFT-Firmen) für ungefähr $50 \%$ des gesamten Handelsvolumens in Cash- und Futures-Märkten ${ }^{6}$ verantwortlich; andererseits machten die Bankhändler 30-40 \% im Cash-Markt und $20 \%$ im Futures-Markt aus; allerdings ist dieses Ausmass und die Aufteilung der Aktivitäten, so der Report der Kommission, nicht ungewöhnlich. Es wurde aber bemerkt, dass die Beteiligung von HFT-Firmen in den relevanten Minuten rasant wuchs (zwischen 9.33 und 9.39 Uhr auf 73,5\% im Cash-Markt und auf 68,4 \% im Futures-Markt). Wie im Fall des Mai-Crashs 2010 haben auch hier die HFTAktivitäten die Aufmerksamkeit auf sich gezogen.

Im Unterschied zum SEC-Bericht über den Mai-Crash betonte der USTreasury-Report allerdings, dass die High-Frequency-Händler die Anzahl und die Tiefe ihrer Orders insbesondere im Zeitfenster des Mini-Crashs etwas reduziert hatten. Trotzdem haben sie im Laufe des Tages die Orderbuchtiefe sowie die Enge der Spreads garantiert:

«PTFs, as a group, contributed to the order book in a relatively balanced fashion throughout the window, often providing standing bids and offers of approximately similar sizes, though at low absolute levels.» (Treasury 2015, 28)

Gleichzeitig wurden die spezifischen HFT-Aktivitäten unter die Lupe genommen, nämlich eine grosse Zahl von Orderstornierungen sowie das Ausmass des self-trading. Allerdings konnte die Kommission nicht abschliessend beurteilen, ob diese Aktivitäten zu den schnellen Preisbewegungen erheblich beigetragen haben. Klar ist nur, dass die Überflutung des Marktes mit Orders die Zeit der Orderausführung (latency) erhöht hat. In diesem Zusammenhang wies der Report (Treasury 2015, 31) auf die Interdependenzen der Akteure im Markt hin: «As some market participants monitor latency and include it as a variable in their trading strategies, sudden changes in latency would cause them to adjust their behavion», zum Beispiel, aus dem Markt auszusteigen

6 Auf dem Cash-Markt werden Treasury-Anleihen und auf dem Futures-Markt die Futures und Optionen auf die Treasury-Anleihen gehandelt. 
und die Liquidität zu reduzieren. Das self-trading war an dem Tag stark (generierte viele Transaktionen), aber nicht aussergewöhnlich im Vergleich zu «normalen» Handelstagen. Abschliessend hält der Report fest:

«Analysis of participant-level data in the cash and futures markets did not reveal a clear, single cause of the price movement during the event window on October 15. However, the data did highlight a number of important characteristics of the event. They revealed that PTFs remained the dominant participant type during the event window: they produced high trading volumes during the event window, and continued to provide liquidity to the cash and futures order books, though at much reduced levels.» (Treasury 2015, 33; meine Hervorhebung)

Dieses Schlusswort des Reports fasst die wichtigsten Schwierigkeiten zusammen, die generell mit der Beurteilung der Bedeutung von HFT für das Geschehen im Markt verbunden sind. Erstens ist die Ursache-Wirkung-Kette nicht klar. Den Beitrag von HFT-Firmen zu bestimmten Preis- und Liquiditätsentwicklungen zu isolieren, ist sehr schwierig bis unmöglich, weil auch andere Akteure und Faktoren in diesem sehr dynamischen Umfeld mitspielen. Ausserdem verfolgen die HFT-Firmen ganz unterschiedliche Strategien, die wiederum in ganz unterschiedlicher Weise den Markt beeinflussen: Während einige Strategien Liquidität entziehen, gehen andere aktiv in den Markt hinein, generieren hiermit Umsatz und unterstützen die Tiefe des Orderbuchs (OICU-IOSCO 2011, 24).

Deswegen gibt es keine einheitliche Meinung über die Rolle der HFHändler in den Mini-Crashs. Während der SEC-Report über den Crash am 6. Mai 2010 das HFT eher der Verstärkung der Krise beschuldigt, stellt der US-Treasury-Bericht über den 15. Oktober 2014 das HFT tendenziell als heroischen Retter des effizienten, reibungslos funktionierenden Marktes dar, als Fels in der Brandung der enormen Volatilität und der Liquiditätsschwankungen. Der Report betont, dass die HF-Händler hart arbeiten, um diese Funktionen zu erfüllen:

«While PTFs are able to provide very tight bid-ask spreads even in a volatile market, and help ensure that prices rapidly reflect new information, such firms typically do not wish to establish significant positions, and may not have the capital to compete for large buy and/or sell orders. Moreover, unlike dealers, PTFs do not have access to a customer business to assess current and latent demand for liquidity and therefore have to spend considerable effort on processing the information content of incoming order flow.» (Treasury 2015, 39) 
Die zwei Zuschreibungen, die in beiden Reports zu finden sind - einerseits die HF-Firmen als Helden, die die Liquidität zur Verfügung stellen und die Märkte unterstützen, und andererseits als Bösewichte, die die Märkte destabilisieren und zu zerstören drohen - finden sich in den Debatten um den algorithmischen Handel immer wieder.

Ein gutes Beispiel bieten die Diskussionen um den Börsengang von Virtu. Virtu Financial Inc. ist eines der grössten HFT-Unternehmen, das 2015 erfolgreich an die Börse gegangen ist. Interessanterweise war das Initial public offering (IPO) ursprünglich für 2014 geplant, allerdings erschien im März dieses Jahres das Buch Flash Boys von Michel Lewis (2014), in dem behauptet wurde, dass die HFT-Firmen sich auf Kosten von langsameren Marktteilnehmern (zum Beispiel von Asset-Managern) bereichern. Da die öffentliche Meinung sich durch das Buch stark verschlechtert hatte, war Virtu gezwungen, das IPO zu verschieben; ausserdem gab es auch ein gerichtliches Ermittlungsverfahren zu den Handelsstrategien von Virtu, insbesondere zu den Praktiken der latency arbitrage und des front running: Der Generalstaatsanwalt von New York, Eric Schneiderman, sandte ein Schreiben an Virtu mit der Aufforderung, die zweifelhaften Praktiken und die Vereinbarungen mit den Börsen und Handelsplattformen offenzulegen. Allerdings setzte das Unternehmen schon nach einem Jahr seine IPO-Planung fort, wobei es sich eine mediale Verteidigungsstrategie zurechtgelegt hatte, die den positiven Beitrag von Virtu und der HFT-Industrie insgesamt zur Marktstabilität hervorhebt. Auf seiner Website und in dem IPO-Prospekt präsentiert sich Virtu als Market-Maker, der Liquidität im Markt generiert, die Kosten für alle Marktteilnehmer reduziert und dadurch «eine vitale Rolle für die Gesundheit und Effizienz der globalen Finanzmärkte, insbesondere in den turbulenten Zeiten», spielt (www.virtu.com; eigene Übersetzung). Das Unternehmen hat verstanden, dass es sich mit diesen Argumenten als Helden darstellen kann, der den höchsten Idealen der modernen Gesellschaft dient: Marktqualität und Marktstabilität werden als absolute, unbestrittene moralische Werte erachtet. Indem Virtu offiziell die Verantwortung für die Gewährleistung dieser Werte übernimmt, legitimiert es sich und sein Business-Modell. Mit diesen Argumenten, die übrigens das Leitmotiv der gesamten HFT-Lobby widerspiegeln, machte Virtu sein Listing im Jahr 2015 letztendlich zu einem Erfolg.

Durch solche heroischen Selbstinszenierungen der HFT-Firmen und ihrer Lobby lassen sich ihre Gegner natürlich nicht zum Schweigen bringen. Neben dem New Yorker Generalstaatsanwalt Eric Schneiderman sind das zum Beispiel Blogger (z. B. zerohedge.com). Der Zerohedge-Blog kommentierte den Report von US Treasury über den Flash Crash am 15. Oktober 
2014 zynisch und behauptete, dass der Report die Praktiken von HFT gezielt verschönere und heroisiere. Die Blogger sprechen von einer starken HFTLobby, verurteilen die Praktiken der HFT-Firmen (z. B. das quote stuffing) und betrachten sie als grosse Gefahren für die Märkte und die Gesellschaft insgesamt. Der Titel ihres Kommentars zu dem Mini-Crash am 6. Mai 2010 lautet: How HFT Quote Stuffing Caused The Market Crash OfMay 6, And Threatens To Destroy The Entire Market At Any Moment.

Zusammenfassend kann festgestellt werden, dass diese kontroverse gesellschaftliche Debatte sich primär um die Frage nach der Verantwortung für die systemischen Ereignisse im Markt wie mangelnde Liquidität, hohe Volatilität und Mini-Crashs dreht. Diese Ereignisse werden als moralisch relevant angesehen, weil sie das reibungslose Funktionieren der Finanzmärkte beeinträchtigen und daher weitreichende Folgen für die Gesellschaft haben. Dabei gestaltet sich die Verantwortungszuschreibung in beiden Reports als äusserst schwierig und widersprüchlich. Diese Schwierigkeiten möchte ich nun aus der Perspektive der Ethik und der Moralphilosophie analysieren.

\section{HFT und Verantwortung: weitere Überlegungen}

In den oben diskutierten Berichten zeichnet sich die folgende Argumentationskette ab: Man identifiziert ein negatives Event (einen Mini-Crash) und startet die Suche nach den Ursachen dieses Ereignisses. Der Fokus liegt dabei auf den wichtigsten Beteiligten, wie zum Beispiel den HFT-Firmen, die als Subjekte der Verantwortung in Betracht gezogen werden. Das Ziel ist, herauszubekommen, ob ihre Handlungen kausal die Mini-Crashs verursacht haben und ob den HFT-Firmen folglich die Verantwortung dafür zugeschrieben werden kann. Dieser Suche liegt das traditionelle abendländliche Verständnis von Verantwortung zugrunde, das in der Nikomachischen Ethik von Aristoteles seinen Ursprung hat. Es handelt sich um zwei Negativ-Bedingungen für die Zuschreibung von Verantwortung (Coeckelbergh 2012): Erstens dürfen die Akteure nicht gezwnngen sein, etwas zu tun; sie sollen ihre Handlungen und deren Konsequenzen vollständig kontrollieren können (die Kontrollbedingung). Zweitens sollen sie nicht nicht wissen, was sie tun, d. h., es soll für sie möglich sein, die möglichen Konsequenzen ihrer Handlungen voll zu überblicken (die Wissensbedingung). Wenn diese Bedingungen erfüllt sind, so das moderne Konzept der Verantwortung, können bestimmte individuelle Akteure (einzelnen Menschen oder Firmen, die als eine kollektive Einheit betrachtet werden) für verantwortlich gehalten werden. In dieser Denkweise sind auch die Elemente der klassischen Entscheidungstheorie 
deutlich zu erkennen, die die ethischen Debatten seit der zweiten Hälfte des 20. Jahrhunderts stark beeinflussen (Hansson 2010): Man fokussiert auf die möglichen individuellen Handlungen, ihre Konsequenzen und Wahrscheinlichkeiten, die in einer Entscheidungsmatrix erfasst und als den Entscheidern bekannt vorausgesetzt werden. Diese Matrix dient den Akteuren als Entscheidungsgrundlage - und den Philosophen als Grundlage für Verantwortungszuschreibung.

Die starke Bindung des moralischen Status eines Akteurs an die Konsequenzen seiner Handlungen - die Konsequenzen, die er angeblich kennt und kontrolliert - ist allerdings in der modernen Welt (und auch in den Finanzmärkten), wo die komplexen Technologien das Handeln und das Geschehen mitbestimmen, nicht unproblematisch. Im Folgenden soll überprüft werden, inwiefern die beiden Negativ-Bedingungen der Verantwortungszuschreibung in den modernen Finanzmärkten erfüllt sind und wer oder was als Subjekt der moralischen Verantwortung (moral agency) betrachtet werden kann.

Die Berichte über die Mini-Crashs haben gezeigt, dass eine eindeutige Bestimmung der Ursache-Wirkung-Kette im Fall der modernen Finanzmärkte nicht möglich ist: Es konnte kein direkter Zusammenhang zwischen dem Handeln einzelner Akteure (der potenziell Verantwortlichen) und dem Marktgeschehen (dem Objekt der Verantwortung) festgestellt werden. Diese Schwierigkeiten weisen auf das grundlegende Problem der modernen Finanztheorie (und Ökonomie) hin, nämlich auf das Nichtwissen über den Mechanismus, der die Makroebene (Märkte, Crashs) und die Mikroebene (das Verhalten der einzelnen Akteure) verbindet.

Die Finanzmärkte sind grundsätzlich dadurch charakterisiert, dass es keine eindeutige Verbindung zwischen einer Finanztransaktion und ihrem Resultat gibt. Die Finanzmarkttransaktionen sind ihrer Natur nach riskant, d. h., eine Handlung - zum Beispiel der Kauf eines Wertpapiers - kann mehrere Folgen haben: Der Preis des Wertpapiers steigt oder fällt, erwartete Zahlungsströme können ausfallen oder ihr realer Wert kann durch Inflation vermindert werden. Mit anderen Worten: Der (erwartete) Outcome einer Handlung - selbst auf den Märkten ohne HFT - ist nicht sicher, sondern möglich oder mehr oder weniger wahrscheinlich. Diese prinzipielle Unsicherheit auf der Mikroebene wird durch das Zusammenspiel der einzelnen Handlungen auf der Makroebene weiter gesteigert. Dieses Zusammenspiel führt zu den unerwarteten systemischen Effekten wie Mini-Crashs oder Finanzkrisen. Die Wissensbedingung für die Übernahme der Verantwortung für diese Ereignisse ist nicht erfüllt: 
«Systemische Konsequenzen wie eine Finanzkatastrophe können oft von einzelnen Akteuren kaum erkannt bzw. antizipiert werden.» (Heinemann 2014, 222)

Es kann nicht vollständig überblickt werden, wie das individuelle Verhalten auf der Mikroebene zu den Ereignissen auf der Makroebene führt. Dies ist so, weil es dabei nicht um die mechanische Summe der einzelnen Handlungen geht, sondern um die nicht linearen Effekte, die zu den unerwarteten Konsequenzen - wie Herding, Bubbles und Crashs - führen:

«Nonlinear means that when you double the dose of, say, a medication, or when you double the number of employees in a factory, you don't get twice the initial effect, but rather a lot more or a lot less. [...] When the response is plotted on a graf, it does not show as a straight line (dinear)), rather a curve. In such environment, simple causal associations are misplaced; it is hard to see how things work by looking at single parts.» (Taleb 2012, 7; meine Hervorhebung).

Die modernen Konzepte der Verantwortung basieren aber auf der Idee der Kausalität: Das Wissen um die Ursache-Wirkung-Kette bedingt die Möglichkeit der Vorhersage der Ereignisse (Wenn ich das tue, passiert genau dies) und der Kontrolle über die Konsequenzen der Handlungen (Ich kann mit meinem Handeln die erwünschten Outcomes herbeiführen und die unerwünschten vermeiden).

Das Nichtwissen um den kausalen Aggregationsmechanismus von einzelnen Handlungen zu kollektiven Outcomes sowie die Nichtlinearität dieser Aggregation geben Anlass dazu, die Wissens- und Kontrollbedingungen in den modernen hoch technologisierten Finanzmärkten zu hinterfragen. Im Folgenden möchte ich (ausgehend von den Arbeiten von Coeckelbergh (2012; 2015) zeigen, dass diese Bedingungen insbesondere im Fall von HFT nicht erfüllt sind. Die Konsequenzen einer Technologieanwendung können nicht vollständig gewusst sein und kontrolliert werden. Beunza u. a. (2011) rekurrieren in diesem Zusammenhang auf «the risk of fragmented innovation»: In einem Markt, wo der Innovationsgrad hoch ist und die modernen Technologien verbreitet zum Einsatz kommen, ist das Risiko zu bedenken, dass niemand den technischen Stand des Systems vollständig überblickt und versteht; das Handeln der Akteure kann unerwartete negative Konsequenzen zur Folge haben, die Beunza u. a. (2011) als «epistemic accidents» bezeichnen. Die Mini-Crashs in den modernen Finanzmärkten können als «epistemic accidents» (die den «normal accidents» von Perrow [1999] ähneln) betrachtet werden. Der Begriff «epistemic accidents» weist direkt auf das unvollständige Wissen der Marktakteure über die Konsequenzen ihrer Handlungen und die unvollkommene Kontrolle über die Ereignisse hin. 
In den hoch technologisierten Märkten häufen sich die «epistemic accidents», weil die wechselseitige Abhängigkeit der Marktakteure, die Selbstreferenzialität der Märkte und damit die Gefahren von Herding steigen.

Beide Berichte über die Mini-Crashs konstatierten eine erhöhte Ansteckungsgefahr zwischen den Märkten: Man sieht, wie schnell der Crash vom 6. Mai 2010 aus dem Futures-(E-Mini-)Markt auf den Aktienmarkt übergeschwappt ist. Die Preisentwicklungen erhalten durch die schnellen Ansteckungen einen kaskadenartigen und nicht linearen Charakter und werden durch positive und negative Feedbacks verstärkt. Das intensive wechselseitige Beobachten und Nachahmen der Wirtschaftsakteure führt dazu, dass die Märkte zunehmend selbstreferenziell werden: Das heisst, dass Trends sich bestätigen, verstärken und zu Herding führen.

Die prominente Rolle der automatisierten Handelsprogramme in den modernen Märkten trägt besonders zu diesem Anstieg der Selbstreferenzialität des Finanzsystems bei: Die Algorithmen tendieren dazu, in die gleiche Richtung zu «laufen», weil sie a) oft auf der gleichen Idee basieren (z. B. spoofing, flash orders usw.) und auf die Marktsignale in der gleichen Art und Weise reagieren; b) alle Informationen zunächst ungeprüft als «wahre» Informationen verarbeiten; sie sind leicht manipulierbar, weil sie nicht in der Lage sind, Lügen, Manipulationsabsichten oder sprachliche Nuancen zu erkennen. So wurde zum Beispiel beobachtet, dass wenn der Name der berühmten Schauspielerin Anne Hathaway in den Nachrichten erwähnt wurde, die Aktien von Warren Buffets Berkshire-Hathaway-Investmentunternehmen anstiegen: Die HFT-Programme reagieren blind und oft einheitlich auf die Meldungen in den Medien, ohne deren Inhalte zu prüfen (Madrigal 2011).

Ausserdem macht die Priorisierung der extrem schnellen Datenverarbeitung auf Kosten einer gründlichen Informationsauswertung die Algorithmen leicht manipulierbar und anfällig für Fehlinterpretationen und Missverständnisse. Wenn ein Unternehmen zum Beispiel nach dem Berichten von guten Zahlen keinen überoptimistischen Ausblick gibt, interpretieren dies die Computer gelegentlich als Gewinnwarnung. Umgekehrt können Firmen bei einer Ertragsenttäuschung durch eine aggressive Formulierung ihrer Geschäftsprognose eine positive Kursreaktion auslösen, weil die Rechner antizipieren, dass die Unternehmenssituation sich tatsächlich verbessert habe. Diese Blindheit und Naivität der Programme verstärkt die Feedback-Loops und die Tendenz zum Herding.

In diesem Zusammenhang werden die Grenzen von Wissen und Kontrolle in den modernen Finanzmärkten besonders deutlich: 
«With systematic trading strategies multiplying and swelling in size and complexity, and HFT increasingly influential in a range of markets, the risk is that some of these algos interact in unforeseen, dangerous ways when released into the wild. This is already happening, but could worsen as algos become even more widespread. For instance, it is conceivable that, unforeseen, two rival algos somehow undermine or amplify each other, ramping prices up or down at dizzying speeds. They could also lead to an invisible herding effect, with disastrous consequences should one or several investors have to liquidate their positions - something that triggered a «quant meltdown in the summer of $2007 .{ }^{7}$ But this time the speed and impact could be much greater.» (Wigglesworth 2015)

Die zunehmende Intransparenz des Finanzmarktgeschehens und die Tendenz zum Herding zeigen deutlich, dass die Wissens- und die Kontrollbedingung der Verantwortungsübernahme in den modernen hoch technologisierten Finanzmärkten nicht erfüllt sind. Wie steht es aber um die Ansicht, dass man nur den Individuen oder den Kollektivsubjekten (Unternehmen) Verantwortung zuschreiben kann? Wer ist das Subjekt der Verantwortung für die systemischen Risiken in den modernen Finanzmärkten?

\section{Das Problem der moral agency}

Traditionell beziehen sich Ethik und Moralphilosophie auf das individuelle menschliche Verhalten. Der hybride Charakter der modernen Finanzmärkte wirft allerdings die Frage nach der Agency auf: Wessen Aktionen sind von Relevanz für die Zuschreibung der Verantwortung? Kann man Algorithmen als moralische Akteure betrachten? Worin besteht der Unterschied zwischen Menschen und Algorithmen in dieser Hinsicht?

Die Beantwortung dieser Fragen kann an die Diskussion über den ethischen Status von Robotern anschliessen, die in den Engineering Ethics, den Robot Ethics und der Technikphilosophie geführt wird. Diese Diskussionen betreffen allerdings vorwiegend die technologisch stark fortgeschrittenen Artefakte wie zum Beispiel Unmanned Aerial Vehicles (UAV), die im Krieg verwendet werden (Johansson 2011). Sullins (2006) spricht in diesem Fall von «autonomen Robotern», die selbstständig - mit einem minimalen Input vonseiten menschlicher Akteure - agieren. In Bezug auf solche autonomen Roboter entfalten sich interessante Diskussionen: Während ein Lager darauf

7 In einer Augustwoche 2007 hatte eine ganze Reihe von Hedge-Funds, die quantitative Strategien im Markt verfolgten, beispiellose Verluste. So verlor der Goldman-Sachs-Aktienfonds $30 \%$ seines Wertes innerhab dieser Woche? 
besteht, dass Moralität und Verantwortung nur den menschlichen Akteuren zugeschrieben werden können und die Artefakte moralisch neutral sind (Peterson/Spahn 2011), behaupten die Vertreter der Gegenposition, dass autonome Roboter - auf einem bestimmten Level der Abstraktion (Floridi/Sanders 2004) - als moralische Agenten eigenen Rechts betrachtet werden können.

Sullins $(2006,23)$ nennt drei Bedingungen, die notwendig sind, um einen Roboter als eine unabhängige «moral agency» betrachten zu können:

«The first is achieved when the robot is significantly autonomous from any programmers or operators of the machine. The second is when one can analyse or explain the robot's behaviour only by ascribing to it some predisposition or intention to do good or harm. And finally, robot moral agency requires the robot to behave in a way that shows an understanding of responsibility to some other moral agent.» (Meine Hervorhebungen)

Alle drei Bedingungen sind im Fall der HFT-Algorithmen, die eher als Teleroboter bezeichnet werden können (Sullins 2006), nicht erfüllt: Sie treffen einfache Entscheidungen, indem sie programmierte Regeln umsetzen; diese Regeln werden ihnen von den Menschen vorgeschrieben; ausserdem sind es Menschen, die entscheiden, wann und wie ein Algorithmus eingesetzt, getestet, kontrolliert und aus dem Markt genommen wird. Im Fall von Telerobotern, so Sullins $(2006,24)$, ist es klar, dass die Verantwortung auf die Handlungen und Entscheidungen der menschlichen Anwender zurückzuführen ist:

«If a robot is simply a tool, then the morality of the situation resides fully with the users and/or designers of the robot. If we follow this reasoning, then the robot is not a moral agent, at best it is an instrument that advances the moral interests of others.»

Die Rolle der Menschen scheint entscheidend dafür zu sein, zu bestimmen, ob Algorithmen als moralische Akteure betrachtet werden können. Wie sieht es im Fall des HFT genau aus?

In der Tat passt die Definition der Teleroboter auf die HFT-Programme viel besser als der Vergleich mit phantastischen - autonomen und über eine Intention und einen freien Willen verfügenden - Science-Fiction-Kreaturen. Die HFT-Algorithmen sind noch sehr einfach. Sie bestehen aus primitiven Wenn-dann-Regeln, die in der Computersprache programmiert und im Computer ausgeführt werden. Ein selbstständiges Lernen und das unabhängige Agieren der Algorithmen im Markt (und in der Gesellschaft, wie in Robert 
Harris' [2011] Buch The Fear Index beschrieben) ist noch nicht in Sicht. Heutzutage sind die Algorithmen kaum adaptiv und lernen von der eigenen Erfahrung nur sehr eingeschränkt oder gar nicht. Es wird allerdings erwartet, dass sie immer komplexer werden; es gibt schon erste computerisierte Optimierungsmethoden, die adaptive Handelsalgorithmen entwickeln und optimieren, sodass die Computer sich selbstständig programmieren; ihre starke Verbreitung und Anwendung ist aber nach wie vor Zukunftsmusik.

Die Rolle der Menschen für die Existenz und das Funktionieren der HFT-Programme ist zentral - Menschen greifen aktiv an mehreren Stellen in deren Tätigkeit ein. Bainbridge (1983) sprach von der Ironie der Automatisierung: Je fortgeschrittener die Technik, desto stärker ist die Beeinflussung durch den menschlichen Operator. Erstens werden die HFT-Algorithmen von Spezialisten entwickelt und programmiert. Zweitens entscheiden Menschen darüber, wo und wie sie arbeiten. Dies betrifft vor allem menschliche Entscheidungen darüber, wann ein Algorithmus geändert oder abgeschaltet werden soll.

In der Tat setzen sich die HFT-Programmierer ständig mit ihren Algorithmen auseinander. Sie sind gezwungen, ihre Algorithmen häufig zu aktualisieren oder aus dem Markt zu nehmen: Die Lebensdauer eines Algorithmus variiert zwischen einigen Wochen und einigen Monaten. Die Studie von Capgemini (Agarwal 2012) nennt zwei wesentliche Gründe für diese relativ kurze Lebensdauer: Erstens das regelmässige Update der Programme, weil die Marktbedingungen und damit die Annahmen, die in die Algorithmen programmiert sind, sich ändern. So haben die Trader zum Beispiel die Sorge, dass ihre Programme das Ende der Niedrigzinsphase nicht erkennen - sie überlegen sich daher, die relevanten Programme schon früher anzupassen und umzuschreiben (Tages-Anzeiger 2012). Zweitens haben die HFTProgramme eine sehr kurze Lebensdauer, weil die Konkurrenten sie entschlüsseln und nachahmen: Das sogenannte reverse engineering erlaubt ihnen ausgehend von der Beobachtung, was ein fremdes Programm im Markt macht - zu rekonstruieren, wie das Programm beschaffen ist, und es nachzubauen.

Weiterhin werden die Algorithmen hinsichtlich ihrer Performance ständig von Menschen überwacht. In einer ethnografischen Studie beschreiben Lange und Borch (2014) «human monitoring» und generell das Zusammenspiel zwischen den Händlern und ihren Algorithmen als zentrale Komponenten des elektronischen Marktes. Diese Beziehung Händler - Maschine ist sehr intensiv: «One trader noted that $\triangle$ I don't go more than 45 minutes without checking my strategies, that's absolutely sure».» (Lange/Borch 2014, 18) 
Wenn die Performance nicht zufriedenstellend ist, wird der Algorithmus aus dem Markt genommen.

Ein wichtiger weiterer Bereich der menschlichen Einmischung in das Funktionieren der Algorithmen ist ihre Abschaltung, wenn es «brenzlig» wird. Dies passiert heutzutage nicht nur auf der Ebene der individuellen Händler. Unmittelbar nach dem Mini-Crash am 6. Mai 2010 wurde der weltweite Einsatz der sogenannten circuit breakers («Schutzschalten») für individuelle Aktien initiiert: Sie unterbrechen den Handel bestimmter Wertpapieren auf allen Börsen, wenn der Preis sich um mehr als $10 \%$ innerhalb von fünf Minuten verändert (Poirier 2012, 461). Diese Unterbrechung gibt den Beteiligten die Möglichkeit, nachzudenken und die Marktsituation einzuschätzen. Dieses «Zwangsbremsen» kann man mit einer Situation auf der Autobahn vergleichen: Die Verkehrsteilnehmer werden gezwungen, ihr Auto zu verlangsamen und um sich zu schauen, anstatt kopflos weiterzurasen.

Diese starke Beeinflussung der Funktionsweise der Algorithmen durch Menschen legt die Idee nahe, dass ein Mensch sich hinter jedem Algorithmus «versteckt». Dies ist auch organisatorisch gewährleistet:

«Markets usually ask that algorithms be ascribed to a dedicated person, that is, not only a function or an institution, but a human being: depending on the specificities, it can be the Head of Trading or individual trader ID.» (Lenglet 2013, 318)

Die Compliance-Beauftragten und Regulierer wollen genau wissen, wer mit welchem Algorithmus was macht, obwohl dies oft nicht eindeutig festzustellen ist. Diese Zuordnung der Algos zu den Individuen, die sie kontrollieren, ist entscheidend für eine Zuschreibung von Verantwortung:

«[D]ie Beeinflussbarkeit des technischen Systems und damit die Kontrolle über das technische System werden als wesentliche Voraussetzung für die Verantwortungsübernahme angesehen.» (Grote 2009, 154)

Die Anwendung des Verantwortungskonzepts auf Artefakte, die keinen freien Willen haben und über die Konsequenzen ihrer Handlungen nicht reflektieren können, ergibt keinen Sinn (Stensson/Jansson 2014): Algorithmen können nicht als selbstständige moralische Subjekte betrachtet werden, solange Menschen sie vollständig kontrollieren. Es kann sich höchstens um die Verschiebung der Stellen, an denen die menschliche Kontrolle ausgeübt wird, handeln: 
«One [HFT] designer, for instance, drew an analogy between automating markets and manufacturing. Just as replacing three workers by a machine does not eliminate the possibility for human control of the process - control simply moves to the knobs and levers in the machine - neither does automated trading have to mean relinquishing human control. The digital equivalent of knobs in a trading interface may give ever-greater control to the traders, and afford the possibility to use their judgment in an automated setting.» (Beunza u. a. 2011, 25)

Gleichzeitig scheinen diese Überlegungen im HFT-Kontext nicht eindeutig zu sein. Wenn vollständige Kontrolle eine Voraussetzung für die Verantwortungsübernahme ist, stellt sich die Frage, ob die Menschen in der Tat das Handeln ihrer Algorithmen lückenlos und pausenlos kontrollieren können. Es ist zu bedenken, dass die Reaktionszeit der Menschen die Schnelligkeit der Transaktionen, die Computer tätigen, bei weitem unterschreitet: Es ist prinzipiell ausserhalb des menschlichen Fassungsvermögens, die Vorgänge innerhalb einer Nanosekunde zu erfassen (Beddington u. a. 2012). In einer solchen Situation können Computer viel Schaden anrichten, bevor Menschen reagieren. Ein Beispiel hierfür ist in Edgar Perez' (2013) Buch Knightmare on Wall Street. The Rise and Fall of Knight Capital and the Biggest Risk for Financial Markets beschrieben: Am 1. August 2012 schaltete Knight Capital, damals ein Hauptakteur im Bereich des elektronischen Handelns, ein neues Programm frei; dieses Programm begann plötzlich, eine Unmenge falscher Orders auszustellen. Innerhalb von 45 Minuten verlor Knight Capital 440 Millionen Dollar. So viel Zeit haben Menschen gebraucht, um zu verstehen, dass ein Fehler vorliegt, wo er vorliegt, und um ihn schliesslich zu berichtigen (z. B. das Programm abzuschalten).

Dieses Beispiel demonstriert, dass die Algorithmen sich der menschlichen Kontrolle doch (partiell) entziehen: Sie können Fehler machen oder sie «fixieren» die Fehler, die Menschen machen, und übertragen sie unmittelbar in den Markt. Zum Beispiel, wenn ein Mensch eine falsche Taste drückt (the problem of «fat finger»):

«In this case, even though the mistake originates with the trader's 〈fat finger», the algorithm plays a definitive role, in that it does not leave any time for the trader to cancel and modify the instruction: within a second, the order is managed by the automat, thereby closing the space for the interpretation and correction of the course of action. Such a situation would probably not have occurred in a different setting where the trader would have been working the order himself. Mediating the relation between the trader and the market, the algorithm exemplifies the fixation of (mis) behaviours.» (Lenglet 2011, 60) 
Die menschliche Autonomie wird dadurch eingeschränkt. Die unvermeidlichen Fehler, die im Zusammenspiel zwischen Menschen und Technik entstehen, weisen auf die Grenzen der menschlichen Kontrolle hin. Diese Grenzen werden aber oft in den gesellschaftlichen und wissenschaftlichen Debatten über Technik nicht thematisiert und akzeptiert: Der Mensch (und nicht das Werkzeug) wird als alleiniger Verantwortlicher und «Lückenbüsser» (Grote 2009) betrachtet. Wenn die menschliche Kontrolle über Technik lückenhaft ist, ist jedoch gleichzeitig eine der wichtigsten Voraussetzungen für die Verantwortungsübernahme durch Menschen nach Aristoteles nicht erfüllt.

Wir können zusammenfassen: Da ein HFT-Algorithmus nicht ausreichend autonom agieren kann sowie keine Intentionen und keine Vorstellungen von verantwortlichem Handeln hat, kann er kein selbstständiges moralisches Subjekt sein. Nur der Mensch kommt als moral agency infrage, weil er das Handeln der Algorithmen im Wesentlichen bestimmt und kontrolliert. Gleichzeitig ist diese Beeinflussung und Kontrolle nicht vollständig, weil eben die Algorithmen - einmal in den Markt gesetzt - die vorgeschriebenen Regeln mit einer Schnelligkeit umsetzen, die das menschliche Fassungsvermögen übersteigt. Im Umgang der Menschen mit Maschinen gibt es also immer Zonen der Intransparenz, Unsicherheit und Nichtkontrollierbarkeit. Dies macht die Betrachtung der Menschen als alleinige moralische Subjekte (und als ewige «Lückenbüssen») fraglich. Bei der Beantwortung der Frage nach moral agency muss folglich das hybride Zusammenspiel zwischen menschlichen Akteuren und Algorithmen explizit in Betracht gezogen werden.

\section{Die Verdrängung der informellen Regeln}

In den hybriden Märkten findet ein wichtiger Prozess statt, der die Häufung der «epistemic incidents» und die steigende Instabilität der Märkte stark beeinflusst. Obwohl Menschen nach wie vor das Sagen an den Märkten behalten, werden die zwischenmenschlichen Beziehungen zunehmend aus den Märkten verdrängt. Darum spielen die informellen (ungeschriebenen) Regeln eine immer geringere Rolle, obwohl sie - wie die Forschung zeigt - essenziell für das reibungslose Funktionieren und die Stabilität der Märkte sind. Sie sind ein wichtiger Teil des sozialen Mechanismus des Markterhalts: In einem Markt, wo die Händler einander persönlich kennen, fühlen sie sich oft moralisch verpflichtet, Liquidität bereitzustellen oder engere Spreads anzubieten (Abolafia 1996). Baker (1984) hat demonstriert, wie Market-Makers sich an eine informelle Norm halten, nicht zu verkaufen, wenn Preise fallen. Demnach sind die Liquidität und das reibungslose Funktionieren der Märkte - 
auch in schwierigen Zeiten - nicht das Resultat einer rigorosen Regulierung oder offizieller Verhaltenskodizes, sondern der Einhaltung schwacher informeller Regeln.

Genau an dieser Stelle haben die HFT die Natur der Märkte radikal verändert: Obwohl sie, wie oben diskutiert, die Menschen aus den Märkten nicht verdrängen, machen sie die Märkte anonymer. Coeckelbergh (2015) spricht von moral distancing: Menschen verschwinden hinter den Bildschirmen; persönliche Kontakte werden für den Erfolg in dem automatisierten Markt weniger wichtig. Algorithmen handeln untereinander ohne Rücksicht auf Fairness, Reziprozität - und ohne Sorge um den Markterhalt. Hiermit erschüttert HFT eine der zentralen Säulen, auf denen die Marktstabilität beruht, und erhöht die systemischen Risiken.

\section{Zwischenfazit: die Schwierigkeiten der Verantwortungszuschreibung}

Die Überlegungen in den vorigen zwei Abschnitten legen nahe, dass die Frage nach Verantwortung für die systemischen Risiken in den modernen Finanzmärkten nicht trivial zu beantworten ist. Einerseits liegt das an der veränderten Natur der modernen Finanzmärkte aufgrund ihrer Technologisierung: Die ansteigenden wechselseitigen Abhängigkeiten der Akteure, die Selbstreferenzialität der Märkte und Herding-Tendenzen führen dazu, dass das Wissen über die Konsequenzen der individuellen Handlungen nicht vollständig ist sowie die Kontrolle über diese Konsequenzen nicht lückenlos gewährleistet werden kann; hiermit sind die Voraussetzungen für die Übernahme und Zuschreibung von Verantwortung gemäss traditionellen Konzepten nicht erfüllt.

Gleichzeitig haben die Schwierigkeiten der Zuschreibung der Verantwortung für die systemischen Risiken in den Finanzmärkten auch mit der Spezifik dieser Risiken selbst zu tun. Ihre wichtigsten Charakteristika sind:

a) Sie werden nicht von einer einzelnen Person verursacht.

b) Sie resultieren aus einem nur sehr indirekt koordinierten Verhalten: Die Akteure agieren im Framework eines Systems (zum Beispiel im Finanzmarkt) und tendieren oft zu ähnlichen Transaktionen (sie kaufen oder verkaufen die gleichen Wertpapiere), haben aber keine gemeinsame Intention, dies zu tun, und sprechen sich normalerweise nicht ab; es handelt sich um eine ungewollte (unbeabsichtigte) Koordination, die feedback loops und die Verstärkung von Trends verursacht. 
c) Keine individuelle (einzelne) Handlung verändert die Wahrscheinlichkeit, dass ein Schaden wirklich eintritt; das heisst, wenn ein Akteur entscheidet, aus dem Markt auszusteigen, wird das systemische Risiko dennoch bestehen bleiben (James 2017).

d) Grundsätzlich gibt es Schwierigkeiten, den Beitrag einer individuellen Handlung zu den gemeinsamen Konsequenzen zu isolieren. In der Philosophie wird dieses Problem oft als «problem of many hands» (Thompson 1980) diskutiert. Mit anderen Worten: Es gibt keinen eindeutigen nachweisbaren Zusammenhang zwischen den Handlungen der individuellen Akteure und ihren Handlungsfolgen.

e) Falls systemische Risiken eintreten, könnte das gesamte System gefährdet werden; hiermit sind die Verursacher oft auch die Betroffenen der Katastrophe.

f) Systemische Risiken sind schwer einzuschätzen, weil das katastrophale Ereignis meistens aus komplexen Ursache-Wirkung-Ketten resultiert (Renn/Keil 2008) und daher oft nicht vorhersehbar ist; wie Heinemann $(2014,195)$ zurecht anmerkt, handelt es sich dabei eher um «systemische Unsicherheiten».

g) Die möglichen prospektiven (künftigen, nicht vergangenen) Ereignisse sind im Fokus. Bis jetzt hat sich die Moralphilosophie primär auf die retrospektive Verantwortung fokussiert.

Angesichts dieser Charakteristika systemischer Risiken wird die Frage nach der Verantwortung zu einer besonderen Herausforderung. In der ethischen Literatur werden diese Herausforderungen als «responsibility gap» (Owen u. a. 2013), das Problem der «Delusion» (Heinemann 2014) oder «organisierte Unverantwortlichkeit» (Beck 1988) diskutiert.

Beck $(1988,104)$ definierte das Problem «der organisierten Unverantwortlichkeit» als «Widerspruch zwischen systemimmanent erzeugten und systemimmanent nicht zurechenbaren, nicht verantwortbaren, nicht bearbeitbaren Gefahren». Insbesondere wenn es um die Verantwortung für die künftigen Ereignisse geht, überkommen einen Zweifel, ob man in so einer Situation überhaupt noch von Verantwortung reden kann:

«There is also room for sceptical worries about the very existence of shared prospective moral responsibility. Focusing on the fact that an agent really cannot make a difference to an outcome given what others do, we might lose the sense that the agent shares responsibility for ensuring or preventing that outcome. This suggests that intuitions of shared prospective responsibility without individual 
control might be illusory, evaporating when we pay sufficient attention to detail.» (Björnsson 2011b)

Diese Überlegungen machen deutlich, dass die traditionellen, auf den aristotelischen Bedingungen begründeten Verantwortungskonzepte nicht unmittelbar funktionieren. Man müsste sich auf die Suche nach neuen Ideen begeben, um dem Problem der Verantwortung für die systemischen Risiken im Markt beizukommen. Es müssten Konzepte sein, die die Unvollständigkeit des Wissens der Akteure, Grenzen der Kontrollierbarkeit der Technik, die hybride Natur der modernen Finanzmärkte sowie den nicht intendierten Charakter der kollektiven Outcomes in Betracht ziehen. Im Folgenden begebe ich mich auf die Suche. Zuerst stelle ich ein Konzept der retrospektiven moralischen Verantwortung vor, nämlich den Ansatz von «joint responsibility without individual control〉 von Björnsson (2011a, 181), der einen guten Ausgangspunkt für die weiterführenden Überlegungen zum «problem of many hands» darstellt. Anschliessend werde ich die Idee der distributed responsibility diskutieren, die an das Konzept von Tragödie und Verantwortung bei Kierkegaard anknüpft (Coeckelbergh 2012). Diese Ideen werden auf die Fälle prospektiver Verantwortung, systemischer Risiken und automatisierter Märkte angewandt.

\section{Verantwortung für die systemischen Risiken in den modernen Finanzmärkten}

Beide Reports über die Mini-Crashs stellen die Frage nach den Verantwortlichen für die negativen Events, die schon geschehen sind: Waren die High Frequency Traders für die Mini-Crashs verantwortlich? Das ist die klassische Frage, die in der Philosophie als «retrospective outcome responsibility» behandelt wird. Wie schon erwähnt, wird das Problem, dass ein Outcome nicht durch eine isolierte individuelle Handlung verursacht wird, oft dadurch gelöst, dass man eine gemeinsame Aktion («joint action») oder eine gemeinsame Verantwortung, zum Beispiel eines Unternehmens als moralischer Einheit (French 1984), annimmt und analysiert. Man konzipiert ein moralisches Subjekt, das seine Handlungen intendiert, ausführt und kontrolliert - und die intendierten Effekte herbeiführt, für die es dann verantwortlich gehalten wird. Allerdings interessieren wir uns hier für die Fälle, wo der Schaden (oder der Erfolg) gemeinsam bedingt, aber nicht gemeinsam intendiert ist. Deswegen greifen hier die traditionellen Konzepte der outcome responsibility, die auf der Idee der «gemeinsamen Aktion» («joint action») oder «joint agency» (eines Unternehmens) basieren, nicht. High Frequency Traders (und die anderen Marktteilnehmer) bilden kein Kollektivsund haben keine gemeinsamen Ziele, die 
sie zusammen zu erreichen versuchen. Anders gesagt, der Ursprung ihrer gemeinsamen Verantwortung ist nicht in der Zugehörigkeit zu einer bestimmten sozialen Gruppe oder einem Kollektiv zu suchen. Da der Beitrag jedes einzelnen Beteiligten nicht ausreichend ist, um den Effekt (z. B. Mini-Crash) alleine herbeizuführen, können Konzepte von «difference making» und «causal involvement» ebenfalls nicht angewandt werden. Laut diesen Konzepten ist ein Individuum verantwortlich für einen bestimmten Outcome, wenn seine Handlung diesen Outcome unmittelbar und nachweislich verursacht, sodass sein individueller Beitrag wirklich einen Unterschied (difference) macht. Das heisst, ohne diese bestimmte individuelle Handlung wäre es nicht zu dem Outcome gekommen, was im Fall systemischer Risiken nicht gewährleistet ist: Systemische Risiken bleiben bestehen, auch wenn ein Individuum oder einige Individuen nicht handeln.

Vor dem Hintergrund dieser Überlegungen scheint der Ansatz von Björnsson (2011a), der ein Konzept von «joint responsibility without individual control» entwickelt hat, passender zu sein. Sein Konzept hilft, das Problem der Verantwortung für die gemeinsamen Outcomes, die allerdings nicht gemeinsam intendiert und kontrolliert sind, zu strukturieren und zu analysieren.

Björnsson behandelt eine besondere Klasse von Fällen, bei der es um retrospektive moralische Verantwortung für einen bestimmten katastrophalen Outcome geht. Um seinen Ansatz zu illustrieren, benutzt er das Beispiel von drei Bootsbesitzern, die einen See verseucht haben (eigene Wiedergabe):

Jeder von drei Menschen - Alice, Bill und Cecil - hat ein Boot auf einem See. Im Frühjahr hat jeder sein Boot gestrichen und das übrig gebliebene Lösungsmittel in den See gekippt, ohne Wissen der je anderen. Jedem war allerdings bewusst, dass er/sie dadurch das Wildleben im See negativ beeinflusst; jeder der drei ging aber davon aus, dass die Menge zu gering sei, um wirklich (isoliert genommen) einen Schaden anzurichten. Durch die Summierung der Lösungsmittel aller drei stieg die Konzentration im See so stark an, dass die Fische ausstarben. Das Lösungsmittel von einem der drei Akteure hätte den Fischen nicht geschadet, von zweien von ihnen aber schon.

Björnsson analysiert die Frage, inwiefern jedes der drei Individuen für den Outcome (das Aussterben der Fische) verantwortlich ist; er fragt also nach der individuellen Verantwortung. Ähnlich könnten wir die Frage stellen, inwiefern ein einzelner High-Frequency-Händler für einen Mini-Crash verantwortlich sein kann. Die Situationen ähneln einander insofern, als sowohl die drei Bootsbesitzer als auch die HF-Trader einen Effekt herbeiführen, über den sie keine individuelle Kontrolle haben: Der Schaden tritt nur dann ein, wenn 
auch die anderen (oder einige von ihnen) das Lösungsmittel in den See kippen bzw. dem Markt die Liquidität entziehen. Der individuelle Beitrag zu dem finalen Outcome ist in beiden Fällen weder hinreichend noch notwendig. Hiermit fragen wir nach der individuellen Verantwortung in den Situationen, wo es um die gemeinsame (kollektive) Verantwortung geht:

«[T] he responsibility attributed to the three [boot owners; E. S.] is fundamentally collective. Taken together, the faulty behaviours of Alice, Bill and Cecil clearly played a significant causal role in wiping out the fish; individually they did not.» (Björnsson 2011a, 184)

In diesem Sinne sind laut Björnsson die Wähler für den Ausgang eines Referendums sowie Reisende, die häufig ein Auto oder ein Flugzeug benutzen, für den Klimawandel verantwortlich. Dies sind die Situationen, die Coeckelbergh und Wackers (2007) als Situationen der verteilten («distributed») Verantwortung bezeichnen. Sie behaupten, dass insbesondere eine technologisch unterstützte Handlung eine genuin verteilte Handlung ist: Es gibt für sie keinen einzelnen Urheber. Ein Beispiel ist eine Finanztransaktion, die eine ganze Palette von sozialen und moralischen Beziehungen zwischen mehreren Parteien ins Leben ruft. Diese Beziehungen und die damit verbundenen moralischen Verpflichtungen sind nicht festgelegt, sondern kontingent. Jede Transaktion eröffnet jedem Beteiligten einen «Verfügungsraum» - gibt oder entzieht ihm Macht darüber, was geschieht; die Verfügungsgewalt über die Transaktion und ihre Folgen ist daher verteilt über ein Netz von Beteiligten.

Aus dieser Perspektive diskutiert Coeckelbergh (2010) das Beispiel der Explosion der BP-Ölplattform im Golf von Mexiko im Jahr 2010: Normalerweise werden die Manager von BP für den Unfall verantwortlich gemacht, allerdings wurde die Sicherheit der Ölplattformen durch die Zulieferer der technischen Teile, die Bohrunternehmen und die Ingenieure mitbestimmt; auch die Konsumenten, die das billige Öl und Produkte aus Öl massenhaft nachfragen, tragen dazu bei, dass BP ausreichende Investitionen in Sicherheit und Umwelt vermied. Dabei sind diese Verhältnisse nicht festgelegt: Sie entstehen und formieren sich, während man agiert (konstruiert, technische Pläne umsetzt, finanziert, Produkte konsumiert usw.), die Verfügungsmacht erhält und ausübt und hiermit die Verantwortung übernimmt und mit anderen teilt:

«[C]oping with ethical problems related to large and complex technological infrastructures is not only a task for offshore engineers, managers and technicians. 
If many actors have a share in the discretionary power, those actors and the people involved should be held responsible. This includes those who operate on the level of policy, business and finance.» (Coeckelbergh/Wackers 2007, 247)

Ähnlich würde man intuitiv meinen, dass jeder Wähler oder jeder Autofahrer (wie jeder Bootsbesitzer in unserem See-Beispiel) irgendwie verantwortlich für den kollektiven Outcome ist. Für den Fall der Finanzmärkte würde dieses Argument bedeuten, dass alle, die Finanztransaktionen tätigen - alle Händler, die Algorithmen einsetzen oder nicht einsetzen, Regulierer, Portfoliomanager und Bankkunden -, für die systemischen Outcomes (mindestens partiell) verantwortlich sind. Wie könnte man aber diese Intuition der individuellen Verantwortung für die kollektiv herbeigeführten Effekte konzeptionell festhalten?

Björnsson (2011a) behauptet, dass, um über die individuelle Verantwortung für kollektive Outcomes ohne individuelle Kontrolle zu urteilen, die Erklärung der Situation wichtig ist: «Why something happened is at the core of our thinking about moral responsibility.» (Björnsson 2011a, 191) Er führt die «Erklärungshypothese» ein. Ein wichtiges Element dieser Hypothese ist die «motivationale Struktur» der Akteure. Björnsson behauptet, dass die Outcomes durch die Motivationen der Akteure erklärt werden können; damit lässt sich die Frage nach der Verantwortung jedes Einzelnen beantworten. Er definiert diese «motivationale Struktur» allerdings ziemlich vage als priorities, values, preferences, desires, behavioural and emotional habits usw. Im See-Beispiel versteht er die Motivation, die zum Aussterben der Fische geführt hat, als Mangel an Sorgfalt: Alle drei Bootsbesitzer, Alice, Bill und Cecil, zusammen - sowie jeder Einzelne von ihnen - haben sich um die umweltschädlichen Folgen ihrer Handlungen nicht gekümmert. Diese motivationale Struktur ist ein wichtiger Teil der Erklärung des negativen Outcomes (Tod der Fische), aber sie ist es nur, wenn die Motive jedes einzelnen Akteurs zusammen mit den Motiven der anderen berücksichtigt werden:

«This, I have argued, answers the central questions about shared retrospective moral responsibility, understanding such responsibility as (a) implicating the individual moral agents via their motivational structures, without (b) requiring the capacity for individual difference-making.» (Björnsson 2011b)

An dieser Stelle muss man allerdings anmerken, dass sich der Fall der Finanzmärkte an einigen wichtigen Stellen von dem See-Beispiel unterscheidet.

Erstens gibt es im Finanzmarkt bei weitem mehr als drei Akteure, die zudem völlig verschiedene Ziele mit unterschiedlichen Methoden verfolgen 
(Anleger, Spekulanten, Sparer usw.). Dabei wissen alle Beteiligten, dass es weitere Akteure gibt, die im Markt agieren und zum Outcome beitragen; man orientiert sogar das eigene Verhalten am Verhalten der anderen, indem man die Aktionen von anderen (aggregiert im Preis) beobachtet und zu einer Entscheidungskomponente macht. Wichtig ist: Dadurch, dass die Beteiligten im Finanzmarkt selber Elemente des Finanzmarktsystems sind, sind sie gleichzeitig Entscheidende und Betroffene. In Björnssons Fall sind die Beteiligten ausserhalb des Systems See und schaden nicht sich selbst, sondern «nur» den Fischen. Im Finanzmarkt betreffen die negativen kollektiven Outcomes (wie Finanzkrisen) alle Beteiligten; wenn die Märkte kollabieren, ist es für die Börsianer in New York genauso katastrophal wie für Bauern in Spanien.

Zweitens ist im See-Beispiel der Erklärungsmechanismus hinter dem Geschehen relativ eindeutig: Das Lösungsmittel im Seewasser beeinflusst negativ das Wildleben; es ist Alice, Bill und Cecil bekannt; deswegen ist ihre motivationale Struktur - Mangel an Sorgfalt - ein wichtiges Element der Erklärung; jeder von ihnen hat sich nicht genug Sorgen um das Wildleben gemacht (und das Lösungsmittel in den See gekippt), obwohl jeder wusste, dass dieses Verhalten zu negativen Folgen führen könnte. Der Fall der Finanzmärkte ist weniger trivial: Beide oben diskutierten Reports konnten keinen eindeutigen Erklärungsmechanismus für die Mini-Crashs liefern und daher keine eindeutige Aussage über den Beitrag der potenziellen Verantwortlichen machen. Es ist klar, dass die HF-Trader an den Crash-Tagen am Markt sehr aktiv waren; ob ihre Beteiligung für die Mini-Crashs notwendig und signifikant war oder ob sie im Gegenteil sogar stabilisierend wirkte, lässt sich nicht sagen.

Allgemeiner gesprochen: Die systemischen Risiken entstehen aus dem Herdenverhalten der Akteure im Markt; dieses Verhalten wird nicht bewusst intendiert und ist nicht ein Teil der «motivationalen Struktur» der Akteure: Einem, der letztendlich wie viele andere im Markt agiert, kann man nicht vorwerfen, aus Mangel an Sorgfalt zum Entstehen einer Bubble beizutragen oder einen Crash zu präferieren. Während es für einen Bootsbesitzer in Björnssons Beispiel eine verantwortungsvolle Option wäre «nicht zu handeln», d. h. das Lösungsmittel nicht in den See zu kippen, können die Marktteilnehmer nicht nicht handeln - denn dies würde bedeuten, aus dem Markt komplett auszusteigen. Wenn alle das tun würden, gäbe es keinen Markt, da keine Liquidität mehr vorhanden wäre. Es sieht so aus, dass man der Verantwortung für systemische Risiken im Finanzmarkt nicht entkommen kann, sodass das Problem ihrer Zuschreibung sich nicht wirklich stellt: Alle sind verantwortlich 
dafür, was im Markt geschieht (distributed responsibility); die «motivationale Struktur» jedes Einzelnen spielt keine Rolle.

Diese Ansicht befreit von der Notwendigkeit der Suche nach dem einzelnen isolierten Schuldigen; gleichzeitig bleibt es unklar, wo die Verantwortung beginnt und endet. Gemäss diesem Konzept ist es unmöglich, im Markt verantwortungsfrei zu agieren. Jeder, der Finanzmarkttransaktionen tätigt, übernimmt automatisch Verantwortung für die systemischen Risiken und den Erhalt der Märkte, über die er letztendlich keine vollständige Kontrolle hat. Die einzige Option, der Verantwortung zu entkommen, ist, aus dem Markt auszusteigen. Dies würde aber den Markt stark gefährden.

Vor diesem Hintergrund stellt sich die Frage, ob tatsächlich alle Mitglieder der Gesellschaft im gleichen Masse an der Verursachung der systemischen Marktrisiken beteiligt sind. In anderen Worten: Kann man sagen, dass die HF-Trader als eine besondere Gruppe der Marktteilnehmer die Systemrisiken im Wesentlichen herbeiführen und den anderen Marktakteuren und «normalen Bürgern» diese Risiken zumuten? Dies wäre eine wichtige Unterscheidung bei der Beantwortung der Frage nach der Verantwortung für systemische Risiken.

\section{Die Frage nach der Risikozumutung}

In der Einleitung zu diesem Beitrag habe ich erwähnt, dass den systemischen Risiken im Finanzmarkt eine besondere moralische Bedeutung zukommt, weil angenommen wird, dass sie oft den Menschen zugemutet werden, die sie nicht verursachen (Heinemann 2014; Vogl 2012). In der Moralphilosophie wird die Frage nach der Risikozumutung oft am Beispiel des «unfreiwilligen russischen Rouletts» diskutiert: Jemand setzt einer schlafenden Person eine Pistole an den Kopf, dreht die Trommel und schiesst; die Person kommt nicht zu Schaden und erfährt nicht, was geschehen ist. Ist so ein Verhalten ethisch korrekt? (Hansson 2010; James 2017). Nein, sagen die Philosophen, weil nicht nur die Konsequenzen einer Handlung berücksichtigt werden müssen, sondern auch der eigentliche Akt der Risikozumutung. Man kann eine Person nicht nur einem tatsächlichen Schaden, sondern auch einem potenziellen, aber schwerwiegenden Schaden aussetzen und damit eine moralische Grenze überschreiten.

James (2017) übersetzt dieses abstrakte Beispiel in die Geschichte von einem «Normalbürgen», «Working Class Joe», der in der Zeit nach dem Zweiten Weltkrieg stark von der Finanzmarktliberalisierung (billigere Kredite, erschwingliche Konsumgüter usw.) profitiert hat. Joe starb in hohem Alter, 
kurz bevor die grosse Finanzkrise 2008 begann; also hat er nicht mitbekommen, wie seine Ersparnisse und seine Rente in den Finanzmarktturbulenzen «zerpflückt» wurden. Die Regierung und die Regulierer haben aber durch ihre Politik der Finanzmarktliberalisierung Joe (und vor allem seine finanzielle Sicherheit) hohen Risiken ausgesetzt - wie in dem oben beschriebenen fiktiven Fall mit der Pistole.

Für den Fall der Finanzmärkte stellt sich aus dieser Perspektive die Frage, ob die HF-Trader eine Gruppe der Marktteilnehmer darstellen, die der gesamten Gesellschaft «eine Pistole an den Kopf» setzen. In den gesellschaftlichen Debatten werden sie oft als solche dargestellt. Gleichzeitig lassen die Berichte über die Mini-Crashs vermuten, dass die HF-Trader wichtige, aber nicht alleinige Beteiligte am Marktgeschehen an den relevanten Tagen waren; das Ausmass, in dem sie den anderen die Risiken der Crashs zumuteten, lässt sich nicht unmittelbar ermitteln. Weiterhin ist - laut dem Konzept der «verteilten Verantwortung》 - Joe, der seine Ersparnisse am Finanzmarkt (vielleicht sogar in hoch riskanten innovativen Finanzprodukten) angelegt und eventuell einen Pensionsplan gekauft hat, gleichermassen verantwortlich für die Finanzkrise 2008 wie alle anderen, inklusive Regierung und Regulierer.

Vor diesem Hintergrund stellt sich die Frage, ob systemische Risiken ihrer Natur nach vermeidbar sind. Wie James (2017) zu Recht argumentiert, ist die Wahrscheinlichkeit, im Fall des «ungewollten russischen Roulettes» zu schwerwiegendem Schaden zu kommen, mit 1:6 relativ hoch. Die Wahrscheinlichkeit, mit einem Flugzeug oder einem Auto zu verunglücken, ist viel geringer; das entsprechende Risiko entsteht allerdings immer, wenn Autos oder Flugzeuge benutzt werden. Allerdings rechtfertigt die Tatsache, dass die Wahrscheinlichkeit eines «unglücklichen» Outcomes besteht, nicht die Forderung, den Luftverkehr oder das Autofahren zu verbieten. In ähnlicher Weise könnte man argumentieren, dass systemische Risiken auf dem Finanzmarkt unvermeidbar sind - sie werden nicht eliminiert, wenn eine Gruppe der Marktteilnehmer (die HF-Trader) aus dem Markt verbannt wird. Die Frage bleibt aber offen, ob diese Risiken dadurch reduziert werden (die Frage nach dem Ausmass des Beitrags).

Weiterhin haben die Marktteilnehmer keine Alternativen, die ihnen helfen würden, systemische Risiken zu vermeiden. Während ein Autofahrer zum Beispiel sich entscheiden kann, kein Benzin zu verbrauchen, sondern ein Auto mit Elektroantrieb zu fahren, hat ein Finanzmarktteilnehmer praktisch keine Alternative: Entweder handelt er (und trägt hiermit zum systemischen Risiko bei) oder er bleibt dem Markt fern. 
Natürlich könnte man allerdings auch hier die Frage nach dem Ausmass des Markteinflusses und der Verfügungsgewalt sowie dem Grad und Charakter der Beteiligung im Markt für jeden einzelnen Marktteilnehmer stellen. So wird behauptet, dass die HF-Trader, die «gerichtete» Programme verwenden (die Programme, die das Momentum im Markt initiieren oder unterstützen), potenziell stärker zu den systemischen Risiken beitragen; gleichzeitig wird mit dem Momentum in den Märkten seit Ewigkeiten gespielt, und bei weitem nicht alle Momentumstrategien führen zu Katastrophen (sie sind oft sehr kurzlebig; der Trend dreht sich schnell und unerwartet). Vorherzusehen, ob eine Momentumstrategie zu «katastrophalen» oder zu «normalen» Outcomes führt, ist nicht möglich. Generell ist eine klare Differenzierung zwischen «bösen» Marktteilnehmern, die den anderen systemische Risiken zumuten, und «guten», die unter den systemischen Risiken nur leiden, wie die Diskussion oben suggeriert, schwierig.

\section{Tragik der Märkte}

Bleibt dann die Frage nach der Verantwortung für systemische Risiken im modernen Finanzmarkt unbeantwortbar? Konzeptionell könnte an dieser Stelle die interessante Interpretation des Zusammenhangs von Tragödie oder Tragik und moralischer Verantwortung, die Kierkegaard (1843) vorgeschlagen und Coeckelbergh (2012) aufgegriffen hat, hilfreich sein. Die modernen Kulturen, die von Rationalität und Kontrolle besessen sind, haben das Gefühl für das Tragische prinzipiell verloren. Wenn man über Tragik wieder nachdenkt, muss das allerdings nicht heissen, dass man in einen prinzipiellen $\mathrm{Zi}$ vilisationspessimismus à la Nietzsche und Heidegger verfällt. Die Interpretation einer Handlung als tragisch offenbart nach Kierkegaard die ambivalente Natur auch des Moralischen:

«To recognise the tragic is not to accept fate but to recognise that we have to live within the tension between freedom and fate, activity and passivity, control and lack of control.» (Coeckelbergh 2012, 41)

Der Blick auf dieses ständige Dazwischen bringt mit sich die Einsicht in die «unendliche Sanftmut» des Tragischen, die in klarem Kontrast zur «genauen und strengen» Ethik steht, die sich auf Regeln und Vorschriften beruft. Ein Individuum kann in einem komplexen technologischen Kontext nicht für alles verantwortlich gemacht werden, denn nicht alles, was geschieht, entspringt seinem freien Willen, kann seinen Aktivitäten zugeschrieben werden 
oder unterliegt seiner Kontrolle. Aus diesen Überlegungen zieht Coeckelbergh $(2012$, 42) eine wichtige Konsequenz für das Verständnis von Verantwortung:

«In so far as technological, engineering action is «tragic» action (intermediate between activity and passivity), it is appropriate to ascribe responsibility in a more 〈gentle» way since responsibility is not absolute but gradual. [...] Usually it is inappropriate to say that someone is either absolutely responsible or absolutely not responsible. Responsibility with regard to technological experience and action is distributed and is a matter of degree.»

Dieses Konzept bietet Antworten auf das Problem der Ungültigkeit der Kontroll- und Wissensbedingungen im Zusammenhang mit der Zuschreibung von Verantwortung. Die Kontrolle über die Technik - und über das Marktgeschehen - ist nie vollständig; gleichzeitig tragen die Marktteilnehmer zu dem, was geschieht, etwas bei; sie haben ihren Einfluss allerdings nur partiell unter Kontrolle (manchmal mehr, manchmal weniger, manchmal gar nicht). Wie Coeckelbergh $(2012,42)$ richtig anmerkt:

«There is something wrong with the criterion itself if it assumes the possibility of full control since such a condition cannot be fulfilled with regard to human technological action given the tragic nature of this action.»

Das ist auch der Standpunkt von Grote (2009): Anstatt von der vollständigen Kontrolle der Technik auszugehen und die Menschen für alleinige und omnipotente «Lückenbüsser» und «Sündenböcke» zu halten, soll man lieber die Zonen der Unsicherheit, der Nichtkontrollierbarkeit - und hiermit der menschlichen Fehlbarkeit - klar beschreiben und gestalten. Damit sollte die Verantwortungszuschreibung auf der Prämisse der (partiellen) Nichtkontrollierbarkeit der Technologie (und der Märkte) basieren.

Ähnlich ist es um die Wissensbedingung bestellt: Die Unmöglichkeit vollständigen Wissens relativiert die Verantwortungsübernahme und -zuschreibung. Da es sich nur um graduelles oder partielles Wissen handeln kann, kann nur die Frage nach dem Ausmass oder Anteil der individuellen Verantwortung für den unerwünschten gemeinsamen Outcome sinnvoll sein.

Das Tragische besteht dabei darin, dass, auch wenn Motive, Absichten und Bemühungen auf der individuellen Ebene darauf zielen, die negativen Effekte auf der Systemebene zu vermeiden, es trotzdem zu diesen Effekten kommen kann. James $(2017,253)$ nennt dieses Phänomen «innocence» borne of uncertainty»: 
«It is the same kind of innocence that might justify people in failing to cooperate at all, as, for instance, in a stragedy of the commons due to over-use of a vital common resource. Such situations are often analyzed as the result of self-interested optimizing behavior, which entails a non-cooperative equilibrium (as in prisoner's dilemma variations). But the tragedy can arise among morally cautious resource consumers, for simple lack of evidence about the aggregated consequences of the consumption of others. Perhaps each would happily comply with appropriate limits on personal consumption, if they could somehow be publically established. But when expectations aren't established, and no one is in a position to know that his or her use amounts to over-appropriation, given the total consumption of all users, a vital common resource might be quite innocently exhausted, even this tragically brings death to everyone. The impersonal pattern of over-consumption is tragic but not wrongful in any sense that implies liability to criticism for people have conducted themselves. To any such criticism, each could aptly reply, 〈Terrible, yes; but how was I supposed to know?.»»

Im Anschluss generalisiert James (2017, 253, Fussnote) diese Idee:

«[...] as long as (1) moral principles are essentially for self-governance (and so subject to justification according what addressed agents can do, what they can know, and the expected outcomes of their actions, usw.), and (2) the agents in question are not all-knowing and omnipotent, but limited in their epistemic and other agential powers, then it is possible, in principle, for such agents to suffer terrible outcomes in which no one is morally at fault.»

Diese Einsichten in die moralische Tragik der Märkte relativieren doch deutlich die «ewigen» Bemühungen der klaren Verantwortungszuschreibung in den Finanzmärkten und in der Gesellschaft. Ihre Konsequenzen für die Regulierung und Ethik sollten genau überlegt werden.

\section{Fazit}

Der Grundgedanke des modernen Kapitalismus - die Idee der «unsichtbaren Hand» (Smith 1776/1978) oder der «Oikodizee» (Vogl 2012) - besteht darin, dass mehrere autonome («self-governed») individuelle Handlungen im Wettbewerbsprozess ein kollektiv erwünschtes Ergebnis hervorbringen. Die Analyse des gegenwärtigen Geschehens auf den Finanzmärkten veranlasste allerdings Vogl $(2012,174)$ dazu, «das Ende der Oikodizee» zu diagnostizieren:

«Während man lange Zeit darauf setzte, dass die unzuverlässigen Verhaltensweisen von Einzelnen über Marktmechanismen zur Vernunft gebracht werden, 
muss man nun konzedieren, dass Finanzmärkte als Märkte aller Märkte so operieren, dass sie mit rationalen Entscheidungsprozessen systematisch Unvernunft produzieren.»

Die aktuellen Krisen und Crashs bestätigen die Meinung Vogls, dass der friktionslose Mechanismus der Märkte immer häufiger versagt. Die endogenen Kräfte der Märkte in Form von Feedback Loops, Herding und wechselseitiger Abhängigkeit der Akteure wirken eher destabilisierend. Systemische Risiken wurden zum Hauptthema auf dem Finanzmarkt. Ich habe argumentiert, dass die zunehmende Technologisierung der Märkte, insbesondere im Bereich des automatisierten Handels, diese destabilisierenden Tendenzen verstärkt.

Auf das Problem vom «Ende der Oikodizee» gibt es prinzipiell zwei mögliche Antworten, die oft als gegensätzlich diskutiert werden: Regulierung und Ethik. Während die Regulierung als moderne Kultur der accountability Verhaltensnormen von aussen auferlegt, setzt die Ethik auf internalisierte Normen und Regeln.

In dem vorliegenden Beitrag wurde vor allem die ethische Herangehensweise an das Problem der systemischen Risiken analysiert, besonders hinsichtlich der Frage nach der Verantwortung für systemische Risiken im Markt. Diese Frage ist kaum mit der Idee der «unsichtbaren Hand» kompatibel: Wenn der Markt sowieso alles regelt, warum sollen sich individuelle Marktakteure Gedanken darüber machen, zu welchen kollektiven Outcomes ihre autonomen Handlungen führen? Diese «sorglose» Einstellung ist aber nicht pauschal schlecht: Sie befördert Innovationen und das Unternehmertum, denn auf der individuellen Ebene könnte ein Wirtschaftsakteur unendlich viele Risiken eingehen, Neues ausprobieren, ohne sich darum zu kümmern, ob seine Innovation dem Gemeinwohl dienen wird. Das Individuum war durch eine solche Perspektive von den genannten ethischen Sorgen befreit.

Heutzutage wird generell von allen verlangt, sich Gedanken über den individuellen Beitrag zum Gemeinwohl zu machen: Man erwartet etwa, dass, wenn jemand bestimmte Speisen isst oder ein Auto fährt, er oder sie versuchen muss, einzuschätzen, wie stark man dem Klima schadet. Die aktuelle Debatte um den HF-Handel zeigt, dass auch auf den Finanzmärkten erwartet wird, dass die einzelnen Akteure sich prinzipiell darum kümmern, wie stark sie zu den systemischen Risiken beitragen. Es wird argumentiert, dass systemische Risiken moralisch relevant sind: Eine Finanzmarktkatastrophe richtet signifikante Schäden für eine grosse Zahl von Menschen an; deswegen müssen die im ethischen Sinne Verantwortlichen identifiziert werden, die - durch 
verantwortungsvolles Handeln - Finanzkatastrophen verhindern und zum Erhalt des Marktes beitragen sollen (Heinemann 2014). Die HF-Trader scheinen die besten Kandidaten dafür zu sein.

Der vorliegende Beitrag zeigte allerdings am Beispiel des HFT, dass es aus mehreren Gründen schwierig ist, im Finanzmarkt eine eindeutige Verantwortungszuschreibung vorzunehmen. Die Analyse der offiziellen Berichte über die Mini-Crashs macht einerseits deutlich, dass es nicht möglich ist, die HF-Trader auf dem pragmatischen Wege (über ihre prozentuale Teilnahme am Marktgeschehen, ihren Beitrag zu Liquidität und Volatilität usw.) als massgebliche Verantwortliche auszuweisen. Andererseits zeigen die moralphilosophischen Konzepte, die sich mit der individuellen Verantwortung für die kollektiven Outcomes beschäftigen, ebenso keinen eindeutigen Weg auf, wie die Zuschreibung der Verantwortung für systemische Risiken im Finanzmarkt erfolgen könnte. So schlägt das vielversprechende Konzept der «motivationalen Struktur» (verstanden zum Beispiel als «Mangel an Sorgfalt») fehl, da unvollständiges Wissen und mangelnde Kontrolle dazu führen können, dass auch die Handlungen «besorgten» Individuen zu katastrophalen Folgen führen (innocence) borne of uncertainty). Die Tragik der Märkte besteht eben darin, dass jeder, der am Markt agiert, zu den systemischen Risiken beiträgt, aber dafür nicht verantwortlich gemacht werden kann. Am anderen Pol der Diskussion wird vorgeschlagen, die Verantwortung am Finanzmarkt als (eher diffus) verteilt anzusehen (distributed responsibility). Es kristallisieren sich aber aus diesen Überlegungen keine eindeutigen Normen und Regeln heraus, denen die Finanzmarktakteure in ihrem individuellen Handeln folgen könnten, um das allgemeine Wohl auf der Makroebene zu befördern. Ich würde behaupten, dass es bis jetzt nicht gelungen ist, einen ethisch relevanten Link zwischen Mikro- und Makroverhalten in den Märkten festzustellen: Wir bleiben konzeptionell bei der «unsichtbaren Hand», die allerdings aus der heutigen Perspektive nicht nur erwünschte, sondern auch katastrophale Outcomes hervorzaubern kann.

Wenn aber die ethische Argumentation nicht weiterführt, ist es kein Wunder, dass die Regulierung in solchen Situationen die Regie übernimmt. Das ist genau das, was wir in den modernen Finanzmärkten beobachten: Die Diskussionen über «moral orders» werden dort von den Aufsichtsbehörden dominiert. Oft wird dieses Verdrängen der Ethik aus den gesellschaftlichen Debatten als negativ empfunden: O’Neill (2002) führt zum Beispiel das Argument an, dass die omnipräsente externe Kontrolle (Regulierung) das Vertrauen auf die schiere Möglichkeit des verantwortungsvollen Handelns in verschiedenen Berufsgruppen unterminiert hat: Das ständige Überwachen 
hat nur eine «Kultur der Verdächtigung» erzeugt und hiermit die Option, dass Vertreter bestimmter Professionen aus sich heraus (autonom) ethisch handeln könnten, so gut wie ausgeschlossen.

Wenn aber die Ethik - wie oben argumentiert - keine einleuchtenden Antworten auf die Probleme des individuellen ethischen Verhaltens und der Verantwortungszuschreibung im Fall systemischer Risiken gibt, erscheint es doch logisch und vernünftig, den Schwerpunkt auf die Regulierung zu legen. Der Vorteil der Regulierung ist, dass sie sich gleichzeitig über die Mikro- und die Makroebene Gedanken machen kann, während Ethik doch zu stark auf die individuelle Ebene fokussiert und im Endeffekt den Link zwischen mikro und makro aus den Augen verliert.

In der Tat beschäftigen sich die Zentralbanken und Finanzmarktaufsichtsbehörden seit der Finanzkrise 2008 - ganz im Sinne vom «Ende der Oikodizee» - mit der Entwicklung von Tools, die helfen könnten, systemische Risiken zu verstehen, zu überwachen und zu vermindern. So propagiert Andrew Haldane (Chefvolkswirt und Executive Director der Bank of England) seit Jahren ein Verständnis des Finanzsystems als eines «Ökosystems», eines eng verflochtenen Netzwerks, und diskutiert zum Beispiel in einem Artikel in Nature (Haldane/May 2011), wie dieses Verständnis - in die Regulierungsansätze übersetzt - zur Reduktion von systemischen Risiken beitragen kann. Die Bundesbank entwickelt einen «makroprudenziellen Ansatz» (www.bundesbank.de), der nicht auf die Stabilität einzelner Banken, sondern auf die Stabilität des gesamten Finanzsystems ausgerichtet ist. Auch international ist das Thema «systemische Risiken» unter den Regulierern sehr prominent: So wurde 2010 von den EU-Mitgliedstaaten der Europäische Ausschuss für Systemrisiken (European Systemic Risk Board, ESRB) mit Sitz in Frankfurt a. M. gegründet, der sich mit der Einschätzung der systemischen Risiken im europäischen Finanzsystem beschäftigt. Damit tragen die Regulierer aktiv zur Generierung von Wissen darüber bei, wie systemische Risiken entstehen, und übersetzen dieses Wissen in Empfehlungen, Normen und Vorschriften (zum Beispiel für Eigenkapital und die Liquidität der Banken).

Der Fokus auf der Regulierung verstärkt allerdings die moderne Illusion der Möglichkeit vollständigen Wissens und vollständiger Kontrolle, von der ich mich in diesem Beitrag eher distanziert habe. Die Regulierung - genauso wie die ihrem Kern nach «strenge und harte» Ethik - ist keine adäquate Antwort auf die hybride Natur und Tragik der modernen Märkte, in denen Wissen und Kontrolle prinzipiell eingeschränkt sind. 
Wichtig ist auch, dass die Regulierer durch die Durchsetzung von formalen Normen und Vorschriften eher zur Instabilität der Märkte beitragen, indem sie die informellen Regeln aus dem Markt verdrängen und hiermit den Effekt, den HFT auf die Märkte hat, noch verstärken. Diesen Prozess hat Macey (2013) an mehreren Beispielen verdeutlicht: In den stark regulierten Ländern und Märkten konnte man in den letzten Jahren einen «Kollaps der Nachfrage nach Reputation» beobachten. Dies geschah unter anderem, weil die Gesetzgebung und Regulierung als Ersatz für Reputation und Reziprozität dienen, die eine Basis für die Entwicklung und das Einhalten informeller Regeln darstellen.

Da die informellen Regeln aber - wie oben diskutiert - zum Erhalt der Märkte beitragen, hat die rigorose und omnipräsente Regulierung, die sie verdrängt, einen ungünstigen Effekt auf der Makroebene. Die adäquate Antwort auf die Tragik der Märkte scheint deswegen ein besonderes Hybrid von Menschen und Maschinen zu sein, das die soziale Struktur der Märkte weitgehend aufrecbterbält. Beunza und Millo (2015) haben gezeigt, dass im New York Stock Exchange eine Struktur besonders erfolgreich war, in der Algorithmen und das traditionelle Börsenparkett koexistierten («blended automation»). Eine Struktur, in der Algorithmen die Menschen aus dem Handel komplett verdrängen, ist tendenziell sehr instabil, da informelle Verantwortung und Reputation aus dem Markt verschwinden würden. Gleichzeitig ist die informelle Verantwortung eine adäquate Antwort auf das Problem des unvollständigen Wissens und der unvollständigen Kontrolle an den Märkten, weil Menschen, die diese Art der Verantwortung übernehmen, die systemischen Risiken gleichzeitig produzieren (indem sie im Markt agieren) und abmildern (über das Einhalten der informellen Regeln). Die informellen Regeln repräsentieren die gesuchte «Bewegung» zwischen Passivität und Aktivität, Schicksal und vollständiger Kontrolle, die für das Konzept von Kierkegaard charakteristisch ist. Aus diesem Grund schlage ich vor, HFT nicht zu verdammen (verbieten), sondern an einer Finanzmarktarchitektur zu arbeiten, die die Co-Existenz von Maschinen und Menschen effizient gestaltet - effizient nicht im Sinne formeller Regulierung oder einer formellen Ethik («streng und hart»), sondern durch den Erhalt der notwendigen sozialen Strukturen, die hinter den - oder durch die - Algorithmen nicht verschwinden dürfen. 


\section{Literatur}

Abolafia, Mitchel Y. (1996): Making Markets: Opportunism and Restraint on Wall Street, Cambridge MA.

Agarwal, Anuj (2012): High Frequency Trading: Evolution and Future, London UK.

Angel, James J. / McCabe, Douglas (2013): Fairness in Financial Markets:

The Case of High Frequency Trading, in: Journal of Business Ethics 112, 585-595.

Bainbridge, Lisanne (1983): Ironies of Automation, in: Automatica 19 (6), 775-779.

Baker, Wayne (1984): The Social Structure of a National Security Market, in: American Journal of Sociology 89 (4), 775-781.

Beck, Ulrich (1988): Gegengifte. Die organisierte Unverantwortlichkeit, Frankfurt a. M.

Beddington, John u. a. (2012): Foresight: The Future of Computer Trading in Financial Markets: An International Perspective, Government Office of Science in UK, www.gov.uk/government/publications/future-of-computertrading-in-financial-markets-an-international-perspective (6.9.2016).

Benos, Evangelos / Sagade, Satchit (2012): High-frequency trading behaviour and its impact on market quality: evidence from the UK equity market. Working Paper Nr. 469, Bank of England, www.bankofengland.co.uk/ research/Documents/workingpapers/2012/wp469.pdf (7.9.2016).

Beunza, Daniel / MacKenzie, Donald / Millo, Yuval / Pardo-Guerra, Juan Pablo (2011): Impersonal Efficiency and the Dangers of a Fully Automated Securities Exchange, Government Office for cience, UK, www.gov.uk/government/publications/computer-trading-impersonalefficiency-and-the-dangers-of-a-fully-automated-securities-exchange (8.9.2016).

Beunza, Daniel / Millo, Yuval (2015): Blended automation: Integrating algorithms on the floor of the New York Stock Exchange (SRC Discussion Paper No. 38). London: Systemic Risk Centre, The London School of Economics and Political Science.

Björnsson, Gunnar (2011a): Joint responsibility without individual control: Applying the explanation hypothesis, in: Vincent, Nicole A. u. a. (Hg.): Moral Responsibility, Dordrecht, 181-199.

Björnsson, Gunnar (2011b): Sharing Responsibility for the Future, conference paper/abstract, www.gu.se/english/research/ publication?publicationId= 151797 (8.9.2016). 
Boatright, John Raymond (2010): Finance Ethics: Critical Issues in Theory and Practice, New York.

Brogaard, Jonathan (2010): High Frequency Trading and Its Impact on Market Quality, http://modernmarketsinitiative.org/wp-content/uploads/ 2013/10/HFT_Trading.pdf (6.9.2016).

Brogaard, Jonathan / Hendershott, Terrence / Riordan, Ryan (2014): High-

Frequency Trading and Price Discovery, in: Review of Financial Studies 27 (8), 2267-2306.

Carrion, Allen (2013): Very fast money: High-frequency trading on the NASDAQ, in: Journal of Financial Markets 16 (4), 680-711.

CFTS/SEC (2010): Findings Regarding the Market Events of May 6, 2010:

Report of the Staffs of the CFTC and SEC to the Joint Advisory Committee on Emerging Regulatory Issues, www.sec.gov/news/studies /2010/ marketevents-report.pdf (8.9.2016).

Cliff, Dave / Brown, Dan / Treleaven, Philip (2011): Technology Trends in the Financial Markets: A 2020 Vision. Driver Review DR3, Foresight Project on the Future of Computer Trading in the Financial Markets, UK Government Office for Science.

Coeckelbergh, Marc / Wackers, Ger (2007): Imagination, Distributed Responsibility and Vulnerable Technological Systems: The Case of Snorre A, in: Science and Engineering Ethics 13, 235-248.

Coeckelbergh, Marc (2010): We're all to blame for the oil spill, in: The Guardian, June 9, www.theguardian.com/commentisfree/cifamerica/ 2010/jun/09/deepwater-horizon-oil-spill-responsibility-bp (8.9.2016).

Coeckelbergh, Marc (2012): Moral Responsibility, Technology, and Experience of the Tragic: From Kierkegaard to Offshore Engineering, in: Science and Engineering Ethics 18, 35-48.

Coeckelbergh, Marc (2015): Money Machines: Electronic Financial Technologies, Distancing, and Responsibility in Global Finance, New York.

Cooper, Ricky / Davis, Michael / Van Vliet, Ben (2016): The Mysterious Ethics of High-Frequency Trading, in: Business Ethics Quarterly 26 (1): 1-22.

Danielsson, Jon / Shin, Hyun Song (2002): Endogenous Risk, www.ucd.ie/ t4cms/danielsson.pdf (7.9.2016).

Danielsson, Jon / Shin Hyun Song / Zigrand Jean-Pierre (2009): Risk Appetite and Endogenous Risk, www.cemfi.es/ftp/pdf/papers/wshop/ riskappetite.pdf (7.9.2016).

Davis, Michael / Kumiega Andrew / van Vliet, Ben (2013): Ethics, Finance, and Automation: A Preliminary Survey of Problems in High Frequency Trading, in: Science and Engineering Ethics 19, 851-874. 
Farrow, Paul (2012): How long does the average share holding last? Just 22 seconds, in: The Telegraph, January 18, www.telegraph.co.uk/finance/ personalfinance/investing/9021946/How-long-does-the-average-shareholding-last-Just-22-seconds.html (7.9.2016).

Floridi, Luciano / Sanders, Jeff W. (2004): On the Morality of Artificial Agents, in: Minds and Machines 14, 349-379.

Fourcade, Marion / Healy, Kieran (2007): Moral Views of Market Society, in: Annual Review of Sociology 33, 285-311.

Future of Life Institute (2016): The Evolution of AI: Can Morality be Programmed?, http:/ futureoflife.org/2016/07/06/ evolution-of-ai (8.9. 2016).

Gilani, Shah (2012): Unless We Act, High-Frequency Trading Will Crash the Markets, http://moneymorning.com/2012/10/16/unless-we-act-highfrequency-trading-will-crash-the-markets (6.9.2016).

Grote, Gudela (2009): Die Grenzen der Kontrollierbarkeit komplexer Systeme, in: Weyer, Johannes / Schulz-Schaeffer, Ingo (Hg.): Management komplexer Systeme: Konzepte für die Bewältigung von Intransparenz, Unsicherheit und Chaos, München, 149-168.

Haldane, Andrew / May, Robert (2011): Systemic risk in Banking Ecosystems, in: Nature 469, 351-355.

Hansson, Sven Ove (2010): The Harmful Influence of Decision Theory on Ethics, in: Ethical Theory and Moral Practice 13 (5), 585-593.

Harris, Robert (2011): The Fear Index, London.

Heinemann, Simone (2011): Systemische Risiken auf Finanzmärkten und das Problem der Verantwortung, https:/ / epub.ub.uni-muenchen.de/12416/1 /Heinemann_Wirtschaftsphilosophie.pdf (7.9.2016).

Heinemann, Simone (2014): Ethik der Finanzmarktrisiken am Bespiel des Finanzderivatehandels, Münster.

James, Aaron (2017): The Distinctive Significance of Systemic Risk, in: Ratio Juris: An International Journal of Jurisprudence and Philosophy of Law 30 (3), 239-258.

Johansson, Linda (2011): Robots and Moral Agency, Theses in Philosophy from the Royal Institute of Technology, Stockholm, www.diva-portal. org/smash/get/diva2:410512/FULLTEXT02.pdf (8.9.2016).

Kierkegaard, Søren (1843): The Ancient Tragical Motif as Reflected in the Modern, in: ders.: Either/or: A Fragment of Life, London, 137-162.

Kirilenko, Andrei A. / Kyle Albert S. / Samadi, Mehrdad / Tuzun, Tugkan (2016): The Flash Crash: High Frequency Trading in an Electronic Market, http://papers.ssrn.com/sol3/papers.cfm?abstract_id=1686004 (7.9.2016). 
Knorr Cetina, K. / Brügger, Urs (2002): Global Microstructures: The Virtual Societies Of Financial Markets, in: American Journal of Sociology 107 (4), 905-950.

Korn, Brian / Bryan Y. M. Tham (2013). Another Flash Crash, www.forbes. com/sites/deborahljacobs/2013/08/09/why-we-could-easily-haveanother-flash-crash/\#259dd1881cdd (21.1.2019).

Lange, Ann-Christine / Borch, Christian (2014): Contagious Markets: On Crowd Psychology and High-Frequency trading. Working paper, Copenhagen Business School.

Lenglet, Marc (2011): Conflicting Codes and Codings: How Algorithmic Trading is Reshaping Financial Regulation, in: Theory, Culture \& Society 28 (6), 44-66.

Lenglet, Marc (2013): Algorithms and the Manufacture of Financial Reality, in: Harvey, Penny u. a. (Hg.): Objects and Materials. London, 312-322.

Lewis, Michael (2014): Flash Boys: A Wall Street Revolt, New York / London. Macey, Jonathan R. (2013): The Death of Corporate Reputation: How Integrity Has Been Destroyed on Wall Street, New Jersey.

MacKenzie, Donald (2016): How Algorithms Interact: Goffman’s «Interaction Order» in Automated Trading. Working paper, University of Edinburgh.

Madrigal, Alexis C. (2011): Does Anne Hathaway News Drive Berkshire Hathaway's Stock?, in: The Atlantic, 18. März, www.theatlantic.com/ technology/archive/2011/03/does-anne-hathaway-news-driveberkshire-hathaways-stock/72661 (24.7.2016).

McNamara, Steven (2016): The Law and Ethics of High-Frequency Trading, in: Minnesota Journal of Law, Science \& Technology 17, 71-152.

Menkveld, Albert J. (2016): The Economics of High Frequency Trading: Taking Stock, in: Annual Review of Financial Economics 8 (1), 1-24.

Minsky, Hyman P. (1970): Financial Instability Revisited: The Economics of Disaster. Prepared for the Steering Committee for the Fundamental Reappraisal of the Discount Mechanism appointed by the Board of Governors of the Federal Reserve System, Washington.

Nagel, Joachim (2012): High Frequency Trading und Marktimplikationen Eine Einschätzung aus Notenbanksicht. Rede anlässlich der TradeTech DACH 2012, www.bundesbank.de/Redaktion/DE/Reden/2012/2012_ 07_04_nagel_hft_und_martkimplikationen.html (7.9.2016).

Nagel, Joachim / Zajonz, Rafael (2012): High Frequency Trading - A Central Bank View, in: Lattermann, Christoph / Loos, Peter / Gomolka Johannes (Hg.): High Frequency Trading: Costs and Benefits in Securities Trading 
and its Necessity of Regulations, BISE-Discussion, http://aisel.aisnet. org $/$ cgi/viewcontent.cgi?article $=1179 \&$ context=bise (7.9.2016).

OICU-IOSCO (2011): Regulatory Issues Raised by the Impact of Technological Changes on Market Integrity and Efficiency: Consultation Report, Technical Committee of the International Organization of Securities Commissions, www.iosco.org/library/pubdocs/pdf/ IOSCOPD 354. pdf (7.9.2016).

O’Neill, Onora (2002): A Question of Trust, Cambridge.

Owen, Richard u. a. (2013): A Framework for responsible innovation, in: Owen, Richard / Bessant, John / Heintz, Maggy (Hg.): Responsible Innovation: Managing the responsible emergence of science and innovation in society, Chichester UK.

Patterson, Scott (2012): Dark Pools: The Rise of the Machine Traders and the Rigging of the U. S. Stock Market, New York.

Perez, Edgar (2013): Knightmare on Wall Steet. The Rise and Fall of Knight Capital and the Biggest Risk for Financial Markets, o. O.

Perrow, Charles (1999): Normal Accidents: Living With High Risk Technologies, Princeton NJ.

Peterson, Martin / Spahn, Andreas (2011): Can Technological Artefacts Be Moral Agents?, in: Science and Engineering Ethics 17, 411-424.

Poirier, Ian (2012): High-Frequency Trading and the Flash Crash: Structural Weakness in the Securities Markets and Proposed Regulatory Responses, in: Hastings Business Law Journal 8 (2), 445-472.

Renn, Ortwin / Keil, Florian (2008): Systemische Risiken: Versuch einer Charakterisierung, in: GAIA 17 (4), 349-354.

Roubini, Nouriel / Mihm, Stephen (2011): Das Ende der Weltwirtschaft und ihre Zukunft. Crisis Economics, München.

Scannell, Kara / Stafford, Philip (2015): Flash crash: Lone trader seen as «a strange character but genius», in: Financial Times, April 24, www.ft.com/ cms/s/2/027fc1ea-ea60-11e4-96ec-00144feab7de.html\#axzz4JZ5Ysiw8 (7.9.2016).

Smith, Adam (1776/1978): Der Wohlstand der Nationen. Eine Untersuchung seiner Ursachen und seiner Natur, München.

Sornette, Didier / Becke, Susanne von der (2011): Crashes and High Frequency Trading. An evaluation of risks posed by high-speed algorithmic trading. Driver Review DR7, Foresight Project on the Future of Computer Trading in Financial Markets, UK Government Office for Science.

Sornette, Didier / Woodard, Ryan (2010): Financial Bubbles, Real Estate bubbles, Derivative Bubbles, and the Financial and Economic Crisis, in: 
Hideki Takayasu, Misako Takayasu, Tsutomu Watanabe (eds.), Econophysics Approaches to Large-Scale Business Data and Financial Crisis, Heidelberg / London / New York, 101-148.

Soros, George (2008). Das Ende der Finanzmärkte - und deren Zukunft.

Die heutige Finanzkrise und was sie bedeutet, München.

Stafford, Philip (2015): Market stress puts focus on high-speed technology, in: Financial Times, October 6, www.ft.com/content/17c58930-685111e5-97d0-1456a776a4f5 (6.9.2016).

Stensson, Patrik / Jansson Anders (2014): Autonomous Technology Sources of Confusion: A Model for Explanation and Prediction of Conceptual Shifts, in: Ergonomics 57 (3), 455-470.

Sullins, John P. (2006): When Is a Robot a Moral Agent?, in: International Review of Information Ethics 6, 23-30.

Taleb, Nassim (2012): Antifragile: Things That Gain From Disorder, New York.

Tages-Anzeiger (2012). Die Krise bringt Supercomputer an ihre Grenzen, 20. August, www.tagesanzeiger.ch/wirtschaft/unternehmen-und-konjunktur /Die-Krise-bringt-Supercomputer-an-ihre-Grenzen/story/15655921 (31.8. 2016).

Thompson, Dennis F. (1980): Moral Responsibility of Public Officials: The Problem of Many Hands, in: The American Political Science Review 74 (4), 905-916.

Treasury (2015): Joint Staff Report: The US Treasury Market on October 15, 2014, www.treasury.gov/press-center/press-releases/Documents/Joint_ Staff_Report_Treasury_10-15-2015.pdf (8.9.2016).

Turbeville, Wallace (2013): Gone In 22 Seconds: How Frequent is High Frequency Trading?, http://prospect.org/article/gone-22-seconds-howfrequent-high-frequency-trading (8.9.2016).

Vogl, Joseph (2012): Das Gespenst des Kapitals, Zürich.

Weller, Brian M. (2016): Efficient Prices at Any Cost: Does Algorithmic Trading Deter Information Acquisition?, http://papers.ssrn.com/sol3/ papers.cfm?abstract_id=2662254 (7.9.2016).

Wigglesworth, Robin (2015): Algo traders, regulators and the age of the «Wall Street» second, in: Financial Times, November 6, www.ft.com/cms/s/0/ 0538655a-840b-11e5-8e80-1574112844fd.html\#axzz4GFi6Psh1 (7.9.2016). 
